FUNCTIONAL RECOVERY OF THE MICROCIRCULATION DURING

SKELETAL MUSCLE REGENERATION

A Dissertation

presented to

the Faculty of the Graduate School

at the University of Missouri-Columbia

In Partial Fulfillment

of the Requirements for the Degree

Doctor of Philosophy

by

CHARMAIN A. FERNANDO

Dr. Steven S. Segal, Dissertation Supervisor

December 2018 
The undersigned, appointed by the dean of the Graduate School, have examined the dissertation entitled

\section{FUNCTIONAL RECOVERY OF THE MICROCIRCULATION DURING SKELETAL MUSCLE REGENERATION}

presented by Charmain A. Fernando, a candidate for the degree of Doctor of Philosophy, and hereby certify that, in their opinion, it is worthy of acceptance.

Professor Steven S. Segal

Professor Virginia H. Huxley

Professor Grzegorz Sowa

Professor Dawn DW Cornelison 


\section{ACKNOWLEDGEMENTS}

I would like to first express my sincere gratitude to my advisor Prof. Steven Segal for the continuous support of my $\mathrm{PhD}$ research, for his motivation, guidance and

immense knowledge. As my teacher and mentor, he has taught me more than I could ever give him credit for here. I would also like to thank each of the members of my dissertation committee for their professional guidance and for teaching me a great deal about both scientific research and life in general. I would also like to say a big thank you to the members of the Segal laboratory for all the support they have provided me with throughout my tenure as a graduate student.

The past few years would have not been possible without the continuous support of my close friends who have been my family away from home, and I am grateful for that from the bottom of my heart. Nobody has been more important to me in the pursuit of this journey than the members of my family, and last but not least I would like to thank my parents and my brother whose unconditional love and guidance are with me in whatever I pursue. 


\section{TABLE OF CONTENTS}

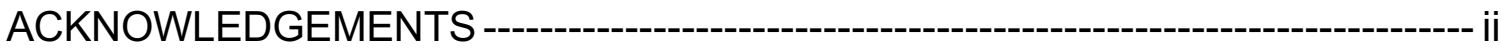

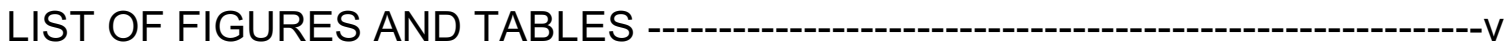

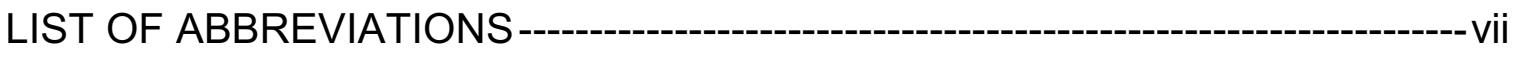

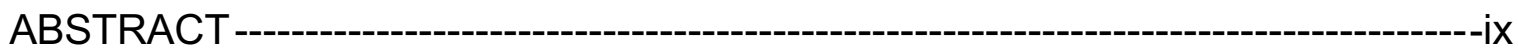

CHAPTER 1 ----------------------------------------------------------------------------------------1

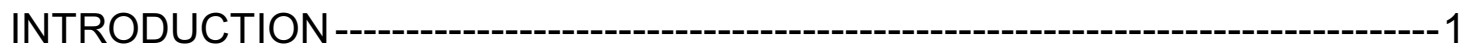

SKELETAL MUSCLE MICROCIRCULATION --_on

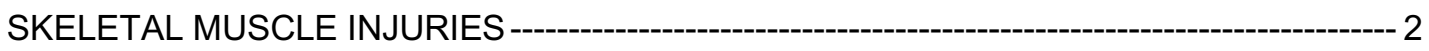

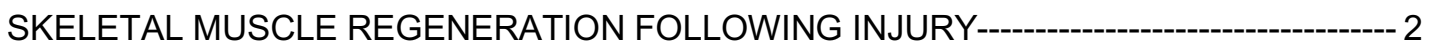

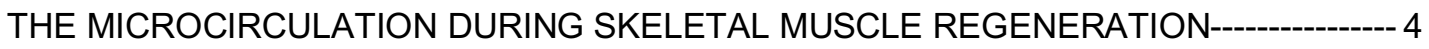

REGULATION OF SKELETAL MUSCLE BLOOD FLOW BY THE RESISTANCE

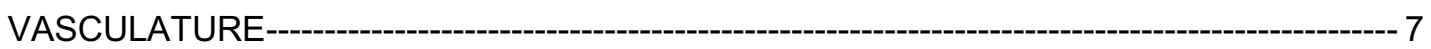

REGULATION OF SKELETAL MUSCLE BLOOD FLOW VIA ENDOTHELIUM-

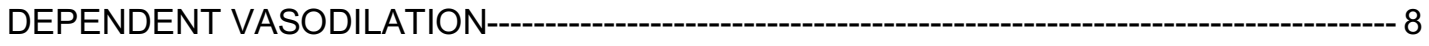

REGULATION OF SKELETAL MUSCLE BLOOD FLOW VIA SYMPATHETIC

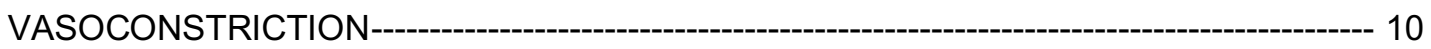

FUNCTIONAL VASODILATION DURING SKELETAL MUSCLE CONTRACTION---------- 11

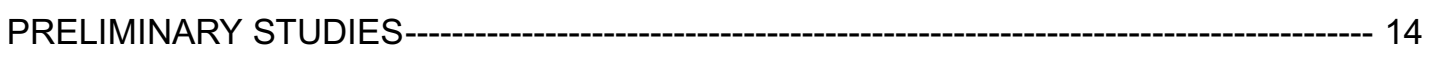

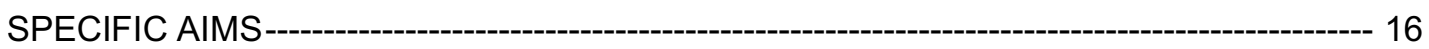

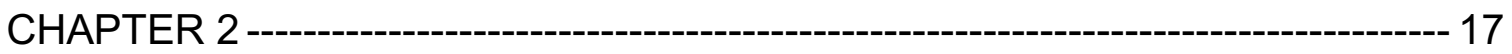

RECOVERY OF VASOMOTOR REACTIVITY AND BLOOD FLOW CONTROL

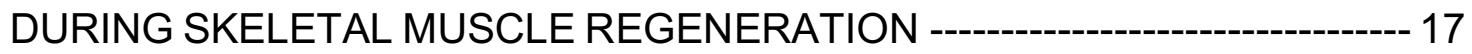

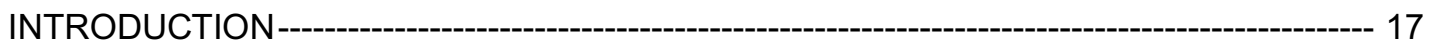

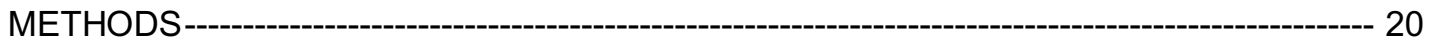




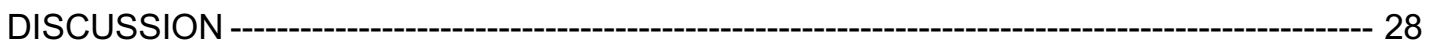

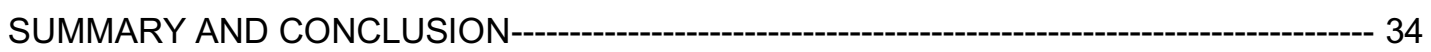

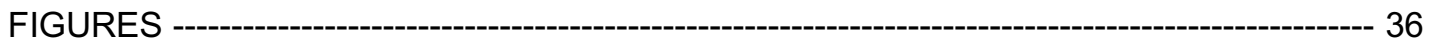

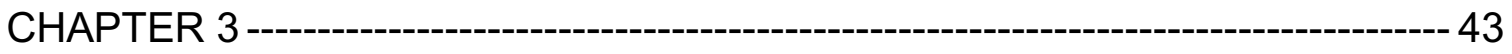

\section{RECOVERY OF FUNCTIONAL VASODILATION DURING SKELETAL}

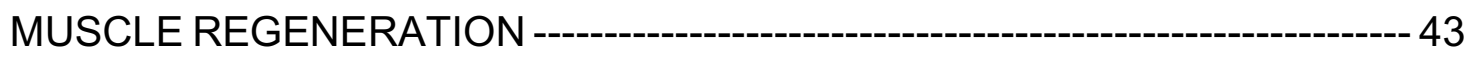

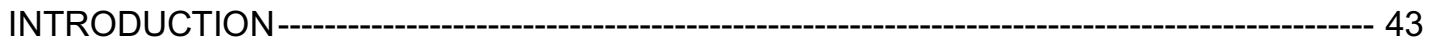

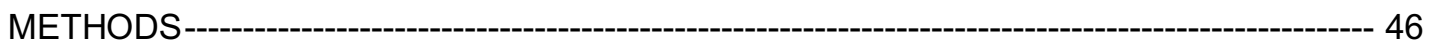

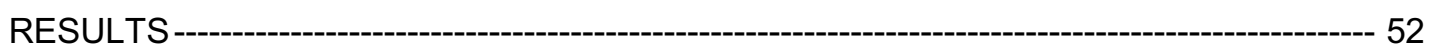

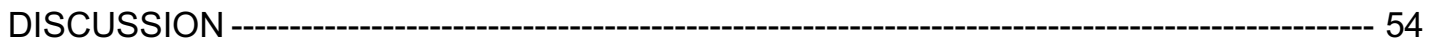

SUMMARY AND CONCLUSION----- 59

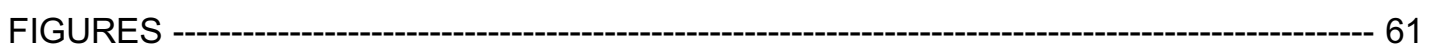

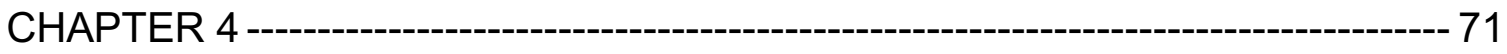

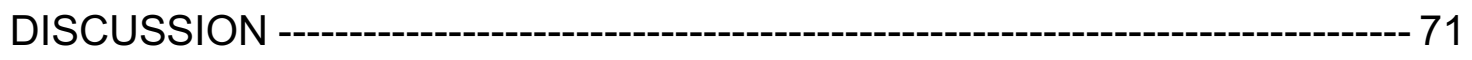

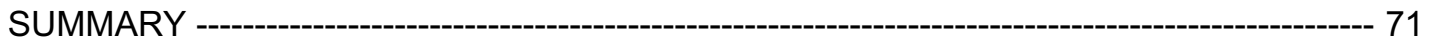

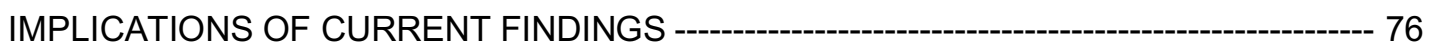

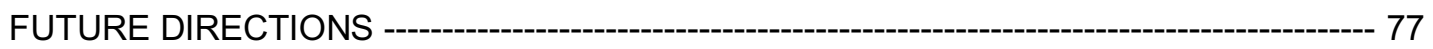

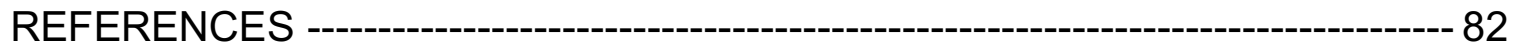

VITA ------------------------------------------------------------------------------------------------ 94 


\section{LIST OF FIGURES AND TABLES}

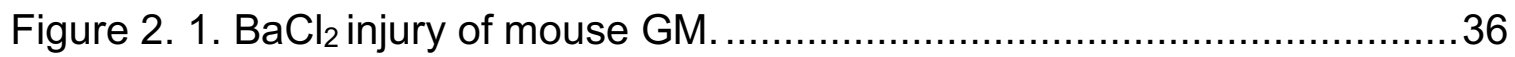

Figure 2. 2. In vivo fluorescence imaging showing microvascular damage with

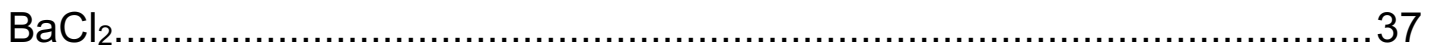

Figure 2. 3. Histology of regenerating skeletal muscle....................................38

Figure 2. 4. GM muscle preparation and branch orders of study.....................39

Figure 2. 5. Resting diameters, maximal diameters and vasomotor tone..........40

Figure 2. 6. Concentration-response curves to ACh. …..................................4 41

Figure 2. 7. Concentration-response curves to PE. ................................... 42

Figure 3. 1. Hematoxylin and eosin staining of uninjured GM muscle fibers (Control) and regenerating GM muscle fibers at 5, 10, 21 and 35d after

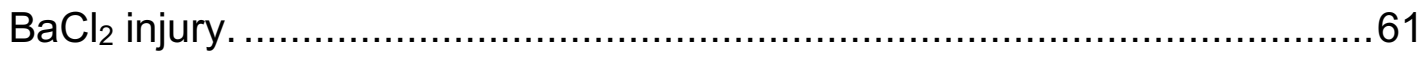

Figure 3. 2. Resting and maximal diameters and vasomotor tone for $F A, 1 A, 2 A$ and $3 \mathrm{~A}$ during regeneration of the $\mathrm{GM}$. .62

Figure 3. 3. Rapid onset vasodilation (ROV) during muscle regeneration:

Diameter change 63

Figure 3. 4. Rapid onset vasodilation (ROV) during muscle regeneration:

Normalized data 64

Figure 3. 5. Time course of Rapid onset vasodilation (ROV) at $500 \mathrm{~ms}$ contraction duration .65

Figure 3. 6. Slow onset vasodilation (SOV) during muscle regeneration: Diameter change 66 
Figure 3. 7. Slow onset vasodilation (SOV) during muscle regeneration:

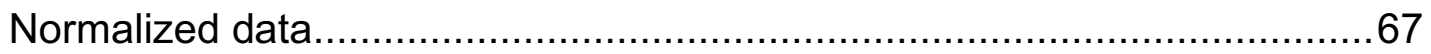

Figure 3. 8. Preparation of the GM for active force measurements...................68

Figure 3. 9 Frequency-force relationships during regeneration of the GM.........70

Table 1. Muscle mass and Cross-Sectional Area (CSA) of the GM during regeneration at $10 \mathrm{~d}, 21 \mathrm{~d}$ and $35 \mathrm{~d}$ compared to Control. ...........................69

Figure 4. 1. Recovery of vasomotor responses and muscle function during

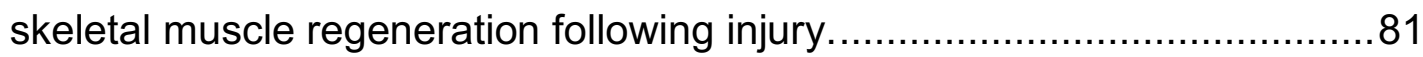




\section{LIST OF ABBREVIATIONS}

$1 \mathrm{~A}: 1^{\text {st }}$ order arteriole

2A: $2^{\text {nd }}$ order arteriole

3A: $3^{\text {rd }}$ order arteriole

ACh: Acetylcholine

$\alpha A R: \alpha$-adrenergic receptors

$\mathrm{BaCl}_{2}$ : Barium Chloride

cAMP: cyclic adenosine monophosphate

cGMP: cyclic guanosine monophosphate

$\mathrm{Ca}^{2+}:$ Calcium

$\left[\mathrm{Ca}^{2+}\right]_{\mathrm{i}}$ : Intracellular calcium concentration

DAG: 1,2-diacylglycerol

EC: Endothelial cell

EDD: Endothelium-dependent vasodilation

EDH: Endothelium-derived hyperpolarization

EDL: Extensor digitorum longus

eMHC: Embryonic myosin heavy chain

eNOS: Endothelial nitric oxide synthase

FA: Feed artery

GM: Gluteus maximus

ID: Internal diameter

$\mathrm{IK}_{\mathrm{ca}}$ : Intermediate $\mathrm{Ca}^{2+}$ activated $\mathrm{K}^{+}$channel

iNOS: Inducible nitric oxide synthase 
$\mathrm{IP}_{3}$ : Inositol 1,4,5-triphosphate

$\mathrm{K}^{+}$: Potassium

$\left[\mathrm{K}^{+}\right]$: Interstitial $\mathrm{K}^{+}$concentration

nNOS: Neuronal nitric oxide synthase

NO: Nitric oxide

NE: Norepinephrine

PE: Phenylephrine

PFA: Paraformaldehyde

PKA: Protein kinase A

PBS: Phosphate-buffered saline

$\mathrm{PGI}_{2}$ : Prostacyclin

ROV: Rapid onset vasodilation

$\mathrm{SK}_{\text {ca: }}$ small $\mathrm{Ca}^{2+}$ activated $\mathrm{K}^{+}$channel

SOV: Slow onset vasodilation

SC: Satellite cell

SMC: Smooth muscle cell

SNP: Sodium nitroprusside

TA: Terminal arteriole

VEGF: Vascular endothelial growth factor

WGA: Wheat germ agglutinin 


\title{
FUNCTIONAL RECOVERY OF THE MICROCIRCULATION DURING SKELETAL MUSCLE REGENERATION
}

Charmain A. Fernando

Dr. Steven S. Segal, Dissertation Supervisor

\begin{abstract}
Skeletal muscle has a remarkable capacity to regenerate following injury, and although muscle regeneration has been studied extensively, little is known about the recovery of the skeletal muscle microcirculation during regeneration. To determine the restoration of blood flow regulation during skeletal muscle regeneration, this dissertation explored the recovery of vasomotor responses to physiological agonists and of functional vasodilation in response to muscle contraction. A novel injury model in the mouse gluteus maximus muscle was developed to study the microcirculation in vivo using intravital microscopy at welldefined time points $(5,10,21$ and 35 days) post injury compared to uninjured Control muscles. Studies encompassed feed arteries and the principal branches $\left(1^{\text {st }}, 2^{\text {nd }}\right.$ and $3^{\text {rd }}$ order $)$ of arteriolar networks comprising the resistance vasculature. Vasomotor responses to agonists and active force developed by muscle fibers recovered by $21 \mathrm{~d}$, however functional vasodilation required $\sim 35 \mathrm{~d}$ to recover. This research provides novel insight into when and to what extent blood flow regulation is restored during skeletal muscle regeneration and provides novel perspective towards developing therapeutic strategies for restoring skeletal muscle function during recovery from injury.
\end{abstract}




\section{CHAPTER 1}

\section{INTRODUCTION}

\section{SKELETAL MUSCLE MICROCIRCULATION}

The role of the microcirculation of skeletal muscle is to supply the myofibers with oxygen and various nutrients and to remove waste products generated during metabolism. The microcirculation begins with a small feed artery (FA) located external to the muscle that branches into arterioles within the muscle. The arterioles are classified based on their branching hierarchy and diameters such that they increase in branch order number as they decrease in diameter. When a FA enters a muscle, it gives rise to first order arterioles $(1 \mathrm{~A})$, and when $1 \mathrm{~A}$ bifurcates, the two daughter branches become second order arterioles $(2 A)(48$, $80,110)$. The number of branch orders depends on the muscle studied and can range from $4 \mathrm{~A}$ to $6 \mathrm{~A}$ before becoming terminal arterioles (TAs). The TAs each give rise to capillary networks. Collecting venules arise from capillaries that merge to become larger venules that transport blood back to the heart. All capillaries (approximately $20-30$ ) perfused by one TA and empty into the collecting venule define the microvascular unit, which is the smallest functional unit for blood flow regulation in skeletal muscle $(19,41)$. The TAs are typically oriented perpendicular to muscle fibers while capillaries run parallel to muscle fibers to maximize the exchange of gas and solutes. Whereas capillaries are composed of a single layer 
of endothelial cells (ECs), the EC (intimal) layer of arterioles and venules is surrounded by a layer smooth muscle cells (SMCs), $(46,61,71,105)$.

\section{SKELETAL MUSCLE INJURIES}

Injury to skeletal muscle can result from a variety of events. For example, blast injuries in armed conflicts and trauma in road traffic accidents account for millions of injuries every year $(89,120)$. In addition, injuries occurring through lacerations, contusions and strains are extremely common in sports $(7,13,27,72)$. Skeletal muscle damage is also observed in degenerative diseases such as muscular dystrophies (38) and occur as a consequence of surgical procedures (e.g., transplantation) involving both cosmetic and functional reconstruction of injured tissue.

\section{SKELETAL MUSCLE REGENERATION FOLLOWING INJURY}

Mammalian skeletal muscle has a remarkable capacity to regenerate following injury, which entails a synchronized process involving the activation of multiple cellular and molecular responses. Regeneration of skeletal muscle occurs in interrelated and time-dependent phases. Although the phases of regeneration are similar between rodent models and humans, the time course is more rapid in rodents and depends on the particular muscle damaged, extent of damage and muscle injury model used $(30,67,75,83)$. 
Injury to skeletal muscle results in rapid necrosis of myofibers, and the myofiber sarcolemma is disrupted resulting in increased permeability (67). Necrosis involves the influx of calcium ions $\left(\mathrm{Ca}^{2+}\right)$, loss of myonuclei and contractile proteins and dissolution of cellular organelles that collectively lead to amorphous debris. These initial events trigger an inflammatory response during the next phase, where neutrophils invade the tissue within approximately 2 hours post injury. The neutrophils release high concentrations of free radicals and proteases, secrete proinflammatory cytokines and recruit monocytes and macrophages $(49,99,118)$. Macrophages increase within 24 hours following injury and are the predominant inflammatory cell type in the damaged region of tissue. Macrophages play a role in removing tissue debris and activating skeletal muscle stem cells, which are known as satellite cells (SCs) $(99,119)$.

The next phase is the regeneration phase. Quiescent SCs reside between the basal lamina and sarcolemma of myofibers and serve as the primary adult stem cell type that becomes activated in response to myofiber injury and are central to the regeneration process $(16,29,37,67,83)$. In response to injury, SCs proliferate and differentiate to give rise to myocytes, which fuse with damaged myofibers for repair or with each other to form multinucleated myotubes for new myofiber formation $(60,67)$. In addition, during the regeneration phase, macrophages change from a pro-inflammatory (M1) phenotype into an anti-inflammatory (M2) phenotype, thereby facilitating myofiber regeneration $(16,29,83,120)$. During the 
remodeling phase, which follows the regeneration phase, there is an overproduction of extracellular matrix proteins; collagens, fibronectin, elastin, proteoglycans and laminin serving to stabilize the tissue and guide the formation of neuromuscular junctions $(67,83)$. The regeneration process is complete when newly regenerated myofibers regain their functional performance and contractile apparatus. In the final phase of regeneration, myofibers become effectively innervated by axons projecting from a-motor neurons in the spinal cord, new neuromuscular junctions are formed and become functional (83).

As summarized through these stages, skeletal muscle regeneration is a complex process that involves multiple cell populations, precise regulation of gene expression, including those for growth factors and of connective tissue components, with each playing an important role in facilitating muscle regeneration and functional recovery following injury $(16,29,67,83,119,120)$.

\section{THE MICROCIRCULATION DURING SKELETAL MUSCLE REGENERATION}

Skeletal muscle injury disrupts the integrity of its microcirculation and the proper structural and functional recovery of these microvessels is necessary to restore muscle function. Microvascular damage with loss of local blood flow due to muscle injury create a hypoxic environment that triggers the generation of new microvessels through the process of angiogenesis in supplying oxygen and nutrients to regenerating myofibers. 
In response to hypoxia, a key pro-angiogenic factor; vascular endothelial growth factor (VEGF) mRNA expression increases and VEGF-A is secreted by myofibers to promote angiogenesis $(9,59,61,71)$. Endothelial tip cells (nonproliferating ECs with filopodia extensions located at the tip of capillary sprouts) contain VEGF receptors such as VEGFR1 (also known as Flt-1) and VEGFR2 (also known as Flk-1) (98) in sensing these VEGF signals, which help guide the nascent EC sprouts towards the angiogenic stimulus. Endothelial stalk cells, which form the trunk of a nascent capillary sprout, proliferate as they follow behind a tip cell, thereby elongating the sprout. When tip cells from two or more capillary sprouts converge at the source of VEGF secretion, they fuse and form a continuous lumen that enables the flow of blood. New capillary formation thereby results in the restoration of muscle perfusion and oxygen delivery. As hypoxia is resolved, VEGF levels decrease along with the stimulus for angiogenesis $(2,53,124)$.

In addition to angiogenesis, muscle injury and resulting ischemia also trigger arteriogenesis, which entails: 1. arterialization (de novo formation of small arteries and arterioles from new or preexisting capillaries) and 2. enlargement of preexisting collateral vessels $(57,90,103)$. Perivascular cells are recruited and differentiate into contractile SMCs during the process of arteriogenesis $(12,90)$. During collateral vessel enlargement, basement membrane degrades, SMCs acquire a proliferative phenotype to increase vessel wall thickness and return to a contractile phenotype when collateral vessel enlargement in complete (57). 
Following ischemic injury in the mouse extensor digitorum longus muscle, regenerated microvascular networks exhibit abnormal morphology including asymmetric arteriolar bifurcations, trifurcations and quadrifications that differ from symmetrical bifurcations characteristic of uninjured skeletal muscle microvasculature (8). Symmetrical arteriolar bifurcations are important to the microcirculation in distributing blood flow evenly to limit hypoxia, thus abnormalities in the structure of regenerated microvasculature may contribute to impaired tissue perfusion. Arteriole-venule shunts, which are typically not observed in healthy muscle, were also observed at locations where arterioles were unable to branch into capillary networks (8). Further, while SMCs are circumferentially wrapped around ECs to form a continuous layer in normal arterioles, the SMC layer of regenerated arterioles were discontinuous and displayed architectural defects (8), which may further contribute to impaired blood flow regulation and skeletal muscle perfusion.

Chronic hypoxic conditions resulting from skeletal muscle injuries can also increase the number of TAs supplying capillaries, as well as increased branching and anastomoses of the arteriolar network along with the development of collateral vessels through angiogenesis and/or arteriogenesis $(8,12,71,91,103)$. Because structural modifications can result in functional changes of the microcirculation, evaluating microvascular function, particularly in branches of the resistance 
network that controls muscle blood flow, is fundamental to understanding the recovery of tissue perfusion during skeletal muscle regeneration following injury.

\section{REGULATION OF SKELETAL MUSCLE BLOOD FLOW BY THE RESISTANCE VASCULATURE}

The resistance vasculature is comprised of small arteries and arterioles that regulate the distribution of blood to tissues of the body according to metabolic demand. For skeletal muscle, small FAs that are located external to the muscle control the volume of blood flowing into arteriolar networks that control blood flow distribution within the muscle and regulate capillary perfusion, where oxygen and nutrients are exchanged with tissue parenchymal cells (68). FAs account for $30-$ $50 \%$ of the total resistance to blood flow, such that perfusion pressure of arterioles is $\sim$ half of systemic arterial pressure, thus FAs are a significant site for blood flow control $(11,68,105)$. Vascular resistance and perfusion pressure (i.e., the difference between arterial and venous pressure) determine tissue blood flow, and because arterial pressure is tightly controlled in the cardiovascular system, vascular resistance is a critical determinant in regulating blood flow. Poiseuille's law:

$$
Q=\frac{\Delta P r^{4} \pi}{8 \eta L}
$$

$(Q=$ flow rate, $\Delta P=$ Pressure difference, $r=$ radius, $\eta=$ fluid viscosity, $L=$ length of tube) explains factors that affect flow rate in a blood vessel. Although vessel length and blood viscosity affect vascular resistance, it is the cumulative changes 
in vessel radii (more typically measured as diameter) that control moment-tomoment changes in vascular resistance as changes in blood viscosity or vessel length are typically negligible. Because blood flow varies with fourth power of the radius, small changes in vessel diameter results in large changes in tissue perfusion (68). Thus, SMCs regulate vessel diameter by either contracting or relaxing to decrease or increase diameter, respectively, and thereby influence the amount of blood flow received by a muscle or particular region of a muscle. In turn, ECs play a major role in relaxing SMCs through the release of vasodilatory substances, and thus indirectly control vessel diameter.

\section{REGULATION OF SKELETAL MUSCLE BLOOD FLOW VIA ENDOTHELIUM- DEPENDENT VASODILATION}

The release of nitric oxide (NO), prostacyclin $\left(\mathrm{PGI}_{2}\right)$ and endothelium-derived hyperpolarization $(E D H)$ leads to SMC relaxation, resulting in vasodilation $(15,34$, $51,68)$. NO is generated from L-arginine via the enzymatic activity of endothelial nitric oxide synthase (eNOS) in ECs, while also generated via the catalyzing effects of inducible NOS (62) and neuronal NOS (nNOS) present in skeletal muscle. This gaseous signaling molecule can then diffuse to the underlying SMC and activate soluble guanylyl cyclase to produce cyclic guanosine-3,5monophosphate (cGMP). cGMP subsequently activates protein kinase $G$, leading to vasodilation by phosphorylating myosin light chain phosphatase, by activating potassium $\left(\mathrm{K}^{+}\right)$channels to cause hyperpolarization, and by the inhibition of $\mathrm{Ca}^{2+}$ 
channels to lower intracellular $\mathrm{Ca}^{2+}$ concentration $\left(\left[\mathrm{Ca}^{2+}\right]_{i}\right)(15,35,114)$. Prostacyclin $\left(\mathrm{PGI}_{2}\right)$ is a potent vasodilator produced from arachidonic acid liberated from membrane phospholipids by phospholipase $A_{2}$. Cyclooxygenase (COX-1 and COX-2) converts arachidonic acid to prostaglandin $\mathrm{H} 2$, which serves as a substrate for the formation of $\mathrm{PGI}_{2}$, via prostacyclin synthase (68). $\mathrm{PGI}_{2}$ then diffuses into SMCs and increases cAMP to activate protein kinase A (PKA), which then phosphorylates and activates ATP sensitive $\mathrm{K}^{+}$channels causing hyperpolarization and relaxation of SMCs $(85,114)$. In addition, an increase in EC $\left[\mathrm{Ca}^{2+}\right]_{i}$ activates small and intermediate $\mathrm{Ca}^{2+}$ activated $\mathrm{K}^{+}$channels $\left(\mathrm{SK}_{\mathrm{Ca}}\right.$ and $\mathrm{IK}_{\mathrm{Ca}}$ ), resulting in the efflux of $\mathrm{K}^{+}$and hyperpolarization of ECs. This hyperpolarization is then conducted into neighboring ECs and into surrounding SMCs via gap junctions to relax SMCs by closing voltage-gated $\mathrm{Ca}^{2+}$ channels $(15$, $34,52)$.

Acetylcholine (ACh) is a neurotransmitter and a potent endothelium-dependent vasodilator that has been used experimentally in evaluating endotheliumdependent dilation (EDD) in larger conduit arteries, small resistance arteries and arterioles $(10,21,48,51)$. The binding of ACh to muscarinic receptors of ECs results in the generation of $\mathrm{NO}, \mathrm{PGI}_{2}$ and $\mathrm{EDH}$ (25). The generation of these vasodilator signaling events in the endothelium collectively lead to relaxation of SMCs resulting in vasodilation and thereby increase skeletal muscle blood flow. 


\section{REGULATION OF SKELETAL MUSCLE BLOOD FLOW VIA SYMPATHETIC VASOCONSTRICTION}

Sympathetic vasoconstriction is integral to regulating vascular resistance and blood flow to skeletal muscle. Sympathetic nerve activity releases norepinephrine (NE) from perivascular sympathetic nerve terminals that acts on $\alpha$ adrenergic receptors (aARs) present on SMCs to cause contraction, resulting in vasoconstriction and restriction of blood flow $(47,78-80)$. Two major aAR subtypes are found in the resistance vasculature: $\alpha_{1}$ and $\alpha_{2}$; both are G-protein coupled receptors. When $\alpha_{1} A R$ is activated, $G_{q}$ protein activates phospholipase $C$ to generate inositol triphosphate $\left(\mathrm{IP}_{3}\right)$ and diacylglycerol $(\mathrm{DAG}) . \mathrm{IP}_{3}$ stimulates $\mathrm{Ca}^{2+}$ release from internal stores and influx through $\mathrm{Ca}^{2+}$ channels located on the plasma membrane, thereby increasing $\left[\mathrm{Ca}^{2+}\right]_{i}$ and activating myosin light chain kinase, with phosphorylation of myosin light chains promoting cross-bridge interaction and SMC contraction. In contrast, when $\alpha_{2} A R$ is activated, $G_{i}$ protein inhibits adenylyl cyclase and decreases the production of cAMP, which increases myosin light chain kinase activity leading to vasoconstriction (43). Vascular SMCs can also express $\beta_{2} A R s$ whereby the activation of this receptor results cytosolic increases in cAMP, PKA and activation of ATP sensitive $\mathrm{K}^{+}$channels resulting in vasodilation $(1,80,85)$.

Studies have shown that the functional distribution of aAR subtypes varies with branch orders in the resistance vasculature $(47,80)$, and that the pattern of subtype distribution among branch orders also varies between different skeletal 
muscles (e.g. gluteus maximus vs cremaster muscle) $(47,80,87)$. In contrast, the functional distribution of $\beta_{2} A R s$ appeared to be uniform in the different branches of the resistance vasculature of the gluteus maximus muscle (GM) (80). Thus, the distribution of $\alpha A R$ subtypes along the resistance vasculature is integral to how sympathetic nerve activity regulates the magnitude and distribution of blood flow to and within skeletal muscle.

\section{FUNCTIONAL VASODILATION DURING SKELETAL MUSCLE CONTRACTION}

With the onset of exercise, increased sympathetic neural activity increases cardiac output and produces vasoconstriction in tissues and organs that are not involved in exercise (e.g. kidney, intestines, non-exercising skeletal muscle) resulting in redistribution of cardiac output to contracting skeletal muscle (68). As a result, skeletal muscle exhibits one of the most dramatic changes in tissue perfusion as blood flow to contracting muscle can increase 50 to 100 -fold to supply the metabolic demands of activity $(28,32,105)$. This increase in blood flow in response to the contractile activity of myofibers is termed functional vasodilation, and the magnitude of functional vasodilation is proportional to the intensity of muscle contractile activity (81).

At the onset of exercise of sufficient intensity there is a concomitant rise in muscle blood flow. This initial hyperemic response occurs within $1-2 \mathrm{~s}$ following a single 
brief contraction and has become recognized as "rapid onset vasodilation" (ROV) $(31,32,36,55,84,100,109,123)$. Although ROV increases with the intensity and duration of a single contraction, the exact mechanism(s) and signaling pathways that mediate ROV are not fully understood. It has been proposed that mechanical factors such as the muscle pump (via compression and relaxation of vessels embedded within myofibers) and vasodilators released from contracting skeletal muscle contribute to $\operatorname{ROV}(31,32,86,101,122)$. One of the main substances associated with ROV is $\mathrm{K}^{+}$that is released from contracting muscle fibers during repolarization from the action potential. Following myofiber contraction, $\mathrm{K}^{+}$diffuses through voltage-dependent $\mathrm{K}^{+}$channels, thereby increasing interstitial $\mathrm{K}^{+}$ concentration $\left(\left[\mathrm{K}^{+}\right]_{\mathrm{o}}\right)$ surrounding the microvasculature. This increase in $\left[\mathrm{K}^{+}\right]_{\mathrm{o}}$ hyperpolarizes SMCs via activation of inward rectifying $\mathrm{K}^{+}$channels and $\mathrm{Na}^{+} / \mathrm{K}^{+}$ ATPase in the plasma membrane $(5,32,55)$. Adenosine produced during muscle contraction may also contribute to ROV. Evidence suggests that adenosine formed on the extracellular side of skeletal muscle cells via membrane bound ecto-5' nucleotidase increases interstitial adenosine concentration. The binding of adenosine to its receptors on SMCs activates CAMP, increases PKA activity, which then phosphorylates $\mathrm{Ca}^{2+}$-activated $\mathrm{K}^{+}$channels to hyperpolarize SMCs and induce vasodilation $(32,97,102)$.

In contrast to ROV, moderate exercise such as walking and cycling, elicit a slower and gradual increase in vasodilation and blood flow which is maintained at a "steady-state" level that is proportional to the tissue metabolic demand. This slower 
rise in vasodilation is termed slow onset vasodilation (SOV) $(20,48,86,112)$. Vasoactive metabolites such as adenosine, hydrogen ion, and lactate produced by the contracting muscle can act to relax SMCs as their concentration increases in the extracellular fluid, while an increase in shear stress acting on the endothelium (e.g. via release of $\mathrm{NO}$ and $\mathrm{PGI}_{2}$ ) further contribute to the progressive dilation of blood vessels during SOV $(32,82,101)$.

Skeletal muscle blood flow is regulated by vasodilation and vasoconstriction through the interaction of SMCs with ECs and sympathetic nerves surrounding the resistance microvasculature. Vasodilatory substances produced in the endothelium contribute to vasodilation, while the release of NE from sympathetic nerves contribute to sympathetic vasoconstriction in regulating blood flow. During exercise, blood flow can increase 50-100 fold in meeting the metabolic demand of muscle fibers, and this increase in blood flow is proportional to the intensity of their contractile activity. Blood flow regulation is impaired as a consequence of skeletal muscle injury and evaluating the extent and time course of recovery of blood flow regulation during skeletal muscle regeneration would provide definitive new insight as to when these mechanisms of vasodilation and vasoconstriction are restored in the microvasculature supplying skeletal muscle. 


\section{PRELIMINARY STUDIES}

Development of GM injury model: To study the microcirculation in regenerating skeletal muscle I developed an injury model in the GM of C57BL/6J mice (4 month old) using $\mathrm{BaCl}_{2}$ (56). In developing a reproducible GM injury, I initially injected different volumes of a $1.2 \% \mathrm{BaCl}_{2}$ solution into the space between the $\mathrm{GM}$ and gluteus medius muscle, creating a "bubble of fluid" in the injection site that injured GM myofibers and its vascular networks. Starting with injection of $25 \mu \mathrm{l}$ and increasing the volume to $50 \mu \mathrm{l}$ resulted in only a partial and inconsistent injury to the GM. Therefore, I increased the volume to $75 \mu$ l and was able to reproducibly damage myofibers surrounding the vascular networks studied. Although the animal survived following unilateral injury to the left GM, bilateral GM injuries using $150 \mu \mathrm{l}$ of $1.2 \%$ $\mathrm{BaCl}_{2}$ was often lethal to the mouse. I therefore decided to conduct unilateral injuries in my criterion experiments. Saline controls $(75 \mu \mathrm{l})$ were performed to evaluate whether the local delivery of fluid by this method resulted in GM damage. I found no damage to the GM following saline injection at 1 day (d), $2 \mathrm{~d}$, and $5 \mathrm{~d}$. Therefore, I utilized uninjured Control (referred to as $0 \mathrm{~d}$ ) to compare with experimental groups at different time points following $\mathrm{BaCl}_{2}$ injury.

Defined time points of study: Tissue histology was performed using hematoxylin and eosin staining at $0 \mathrm{~d}, 1 \mathrm{~d}, 2 \mathrm{~d}, 3 \mathrm{~d}, 4 \mathrm{~d}$ and $5 \mathrm{~d}$ post injury in paraffin-embedded $\mathrm{GM}$ tissue sections (thickness, $5 \mu \mathrm{m}$ ) to determine the timeline for degeneration and necrosis of the $\mathrm{GM}$ with $\mathrm{BaCl}_{2}$ injury. Tissue sections were also stained for hematoxylin and eosin at $10 \mathrm{~d}, 21 \mathrm{~d}$, and $35 \mathrm{~d}$ post injury to determine when new 
myofibers were formed and increased in size. In a separate set of experiments, frozen tissue sections (thickness, $20 \mu \mathrm{m}$ ) were immunolabeled at $5 \mathrm{~d}, 10 \mathrm{~d}$ and $21 \mathrm{~d}$ post injury and in Control for laminin to identify the basal lamina defining muscle cell borders and embryonic myosin heavy chain (eMHC) to label regenerating myofibers, with loss of eMHC indicating maturation of newly formed myofibers. Staining with DAPI identified centrally located nuclei characteristic of regenerating myofibers in contract to nuclei located at the periphery of uninjured control myofibers. I then utilized the histology data to help define key time points for studying the microcirculation during skeletal muscle regeneration.

Contribution to other projects: Development of the novel GM injury model has led to vascular network mapping studies in regenerating skeletal muscle. Thus, to visualize the microvasculature for mapping, I injected fluorescent wheat germ agglutinin (WGA) retro orbitally (129) upon completion of my intravital experiments that day. The WGA binds to N-acetyl-D-glucosamine and sialic acid residues on the surface of ECs, which thereby labels vascular networks and enables visualization of these networks for high resolution mapping of network topology using appropriate image acquisition and analysis instrumentation. My studies have also been used as a reference in utilizing transgenic mice to study skeletal muscle and microvascular regeneration in collaboration with the Cornelison laboratory. 


\section{SPECIFIC AIMS}

The microcirculation is damaged following injury to skeletal muscle, however little is known about the restoration of blood flow regulation during the regeneration process. My working hypothesis is that skeletal muscle regeneration and recovery of contractile function coincide with restoration of the structure and function of its microcirculation. Based upon my preliminary experiments in the mouse GM, the recovery of blood flow regulation is evaluated in vivo at key timepoints: 5 days (d), $10 \mathrm{~d}, 21 \mathrm{~d}$ and $35 \mathrm{~d}$ post injury compared to an uninjured control $(0 d)$.

AIM 1: Evaluate vasomotor control during skeletal muscle regeneration: Sympathetic vasoconstriction and endothelium-depended vasodilation regulate blood flow in skeletal muscle by constriction and relaxation of SMCs, respectively. AIM 1 evaluated when and to what extent vasomotor tone, vasoconstriction and vasodilation recover during regeneration following skeletal muscle injury (CHAPTER 2).

\footnotetext{
AIM 2: Evaluate the coupling between active force production and functional hyperemia during Skm regeneration:

Though integral to physical activity, the ability of the microcirculation to meet the demands of regenerating muscle fibers is unexplored. AIM 2 evaluated when and to what extent the coupling between contractile function and functional hyperemia recover during regeneration following skeletal muscle injury (CHAPTER 3).
} 


\section{CHAPTER 2}

\section{RECOVERY OF VASOMOTOR REACTIVITY AND BLOOD FLOW CONTROL DURING SKELETAL MUSCLE REGENERATION}

\section{INTRODUCTION}

Skeletal muscle is a highly vascularized tissue that comprises approximately $40 \%$ of lean body mass $(64,66,86)$. This dense vasculature participates in metabolic homeostasis by supplying oxygen and nutrients to active muscle fibers and removing the byproducts of metabolism $(71,88,105)$. Traumatic skeletal muscle injuries occur in contact sports, in road traffic accidents, with usage of heavy machinery and in combat $(113,121)$. With injury to muscle, significant damage also occurs in the muscle's vascular supply. Skeletal muscle's remarkable capacity to regenerate following injury has been studied for years $(13,16,30,45,83)$, however, little is known about the recovery of its microcirculation during regeneration of myofibers. In a 1987 study, Burton and Faulkner (24) reported increased resting blood flow with decreased vasomotor tone in regenerating arterioles in the extensor digitorum longus (EDL) muscle grafts in cats. Diminished responses were observed to the vasodilators adenosine and verapamil, and to the vasoconstrictors norepinephrine and $\mathrm{K}^{+}$, leading to the conclusion that the diminished responses were due to abnormalities in the vascular smooth muscle 
layer. In a 2017 study by Arpino et al (8), demonstrated that although a robust angiogenic response is observed after ischemic injury in the mouse EDL, the reconstructed vascular network is flawed in structure with impaired vasomotor control. With little else known about the recovery of the microcirculation after injury, there is a paucity of studies that have evaluated the recovery of vasomotor responses in the microvascular network that regulates blood flow during skeletal muscle regeneration following injury.

Several models of acute skeletal muscle injury have been developed by researchers and offer appealing systems to study interactions between various cell and tissue components in skeletal muscle during regeneration. My goal was to apply such a model to study the microcirculation during recovery following acute injury to myofibers. The gluteus maximus (GM) muscle is an extensor muscle of the hip and, due to its superficial location and overall thinness in the mouse $(\leq 200$ $\mu \mathrm{m})$, makes it well-suited for studying the microcirculation using intravital microscopy $(14,48)$. For my research, I developed a new injury model based on the $\mathrm{GM}$ using $\mathrm{BaCl}_{2}$ to damage its muscle fibers and study the recovery of the microcirculation during myofiber regeneration. Administration of $\mathrm{BaCl}_{2}$ in skeletal muscle causes degeneration and necrosis of myofibers while preserving their resident stem "satellite" cells (SCs) and basement membranes $(26,56)$, which may serve as scaffolds for regenerating myofibers. Quiescent SCs that reside between the basal lamina and sarcolemma of myofibers become activated in response to injury, proliferate and differentiate into myocytes, and these myocytes fuse to form 
myotubes in repairing and replacing damaged myofibers $(16,29,37,56)$. Using intravital imaging and tissue histology, I confirmed that local injection of $\mathrm{BaCl}_{2}$ led to degeneration of myofibers and disruption of their microvasculature. To identify key time points to study the recovery of vasomotor function during regeneration of the GM, I performed fluorescence imaging of fluorescent dyes injected into the circulation to determine the earliest time point for recovery of tissue (capillary) perfusion. My working hypothesis is that recovery of tissue perfusion is critical during the regeneration process for repairing and restoring muscle function. With perfusion restored, evaluating when, where and to what extent vasomotor reactivity and the resulting blood flow control recover during the sequential phases of muscle regeneration defines fundamental questions to be answered by my research.

The resistance vasculature of skeletal muscle, including the GM, begins with small feed arteries (FAs) located external to the muscle and proximal to the microvessels that are embedded in the tissue. Once FAs enter the muscle, they give rise to firstorder arterioles $(1 \mathrm{~A})$ and the branching of $1 \mathrm{As}$ give rise to second-order $(2 \mathrm{~A})$ and third-order arterioles $(3 \mathrm{~A})$, which ultimately branch into the smallest terminal arterioles that give rise to capillary networks oriented parallel to muscle fibers. Capillaries then converge into venules that remove blood from the muscle and carry it back towards the heart $(71,104)$. Proximal branches of the network such as FAs and 1As regulate the amount of blood that enters the muscle, smaller 
downstream branches such as $2 \mathrm{~A}$ and $3 \mathrm{~A}$ regulate flow distribution within the tissue and terminal arterioles regulate capillary perfusion $(61,104)$.

Skeletal muscle blood flow control is a dynamic process whereby multiple stimuli influence the contractile activity of SMCs in resistance vessels either directly or indirectly through ECs $(15,107)$. For example, sympathetic nerve fibers that surround FAs and arterioles release NE and the binding of the neurotransmitter onto $\alpha$ ARs on SMCs causes vasoconstriction $(79,80)$. In contrast, the generation of $\mathrm{NO}$ and hyperpolarization in ECs contribute to $\mathrm{SMC}$ relaxation and vasodilation $(25,47,80)$. The goal of this study was to evaluate when and to what extent vasoconstriction (via activation of $\alpha A R s$ on SMCs) and vasodilation via endothelium-dependent signaling recover during skeletal muscle regeneration. I tested the hypothesis that vasomotor responses recover in parallel with myofiber regeneration.

\section{METHODS}

Barium Chloride-induced skeletal muscle injury: All animal procedures were conducted according to protocols approved by the Animal Care and Use Committee of the University of Missouri, Columbia. Male C57BL/6J mice were obtained from Jackson Laboratory (Bar Harbor, ME, USA) at $~ 3.5$ months of age and acclimated at the University of Missouri animal care facilities at least 1 week 
prior to study. On a given day, a mouse was anesthetized with an intraperitoneal injection of ketamine/xylazine $(100 / 10 \mathrm{mg} / \mathrm{kg})$ in sterile saline. Dorsal skin overlying the GM was shaved, the mouse was placed on an aluminum warming plate to maintain body temperature, and the site was sterilized with Betadine Solution (Purdue Products L.P. Stamford, CT, USA) followed by wiping with $70 \%$ alcohol. A $\sim 5 \mathrm{~mm}$ incision was made through the skin and, using a Hamilton syringe and needle (32 gauge, 0.5 " long, $30^{\circ}$ tip bevel; Reno, NV, USA) $75 \mu \mathrm{l}$ of $1.2 \%$ (w/v) $\mathrm{BaCl}_{2}$ (LabChem, Zelienople, PA, USA) solution was injected into the space between the GM and gluteus medius muscle to injure GM myofibers and microvasculature (Figure 2.1). The incision was closed with VetClose surgical glue (Henry Schein, Dublin, OH, USA) and the mouse was kept warm and monitored closely until it regained consciousness and resumed activity. The mouse was monitored daily for 4 consecutive days to ensure proper healing of the incision.

Time-points of study: Based on my preliminary studies while developing the model, analysis of resistance network branches was conducted in regenerating GM at 5 days (d), 10d and 21d post injury and was compared to an uninjured Control (0d). The selection of these time-points of study was based on recovery of tissue perfusion and histological analyses. To evaluate recovery of tissue perfusion, fluorescein isothiocyanate-dextran (FITC-dextran; $70 \mathrm{kDa}$ to approximate the size of albumin, $2 \mathrm{mg} / \mathrm{ml}$ ) was administered via a retro-orbital injection (129) to label circulating plasma. Tissue cross-sections (20 $\mu \mathrm{m}$ thick) were 
immunolabeled for $\mathrm{eMHC}$ and $\mathrm{DAPI}$ to identify regenerating myofibers and centralized nuclei in newly formed myofibers, respectively.

Surgical procedures: The GM was prepared for intravital microscopy as described previously $(48,63,110)$. Briefly, a mouse was anesthetized with an intraperitoneal injection of pentobarbital sodium $(60 \mathrm{mg} / \mathrm{kg})$. The dorsal skin overlying the GM was shaved to remove hair and the mouse was placed on the warming plate in the prone position to maintain body temperature $\left(37^{\circ} \mathrm{C}\right)$ throughout experiments. While viewing through a stereomicroscope, the overlying skin and connective tissue were removed using microdissection while the exposed GM was continuously superfused with a bicarbonate-buffered physiological salt solution (PSS; $34^{\circ} \mathrm{C}, \mathrm{pH} 7.4$ ) containing (in $\mathrm{mM}$ ) $131.9 \mathrm{NaCl}, 4.7 \mathrm{KCl}, 2 \mathrm{CaCl}_{2}, 1.17$ $\mathrm{MgSO}_{4}$, and $18 \mathrm{NaHCO}_{3}$ equilibrated with $5 \% \mathrm{CO}_{2} / 95 \% \mathrm{~N}_{2}$. The $\mathrm{GM}$ was dissected along the spine from its origins (lumbar fascia, sacrum and iliac crest) and reflected away from the body to expose its vascular supply then spread onto the surface of a transparent rubber pedestal (Sylgard 184; Dow Corning, Midland, MI, USA) and pinned at the edges to approximate in situ dimensions. Supplemental doses (20 $\mathrm{mg} / \mathrm{kg}$ ) of pentobarbital sodium were given throughout experiments to maintain anesthesia. At the end of each day's experiment the mouse was euthanized with an overdose of pentobarbital sodium followed by cervical dislocation.

Intravital microscopy: Upon completion of surgery, the GM preparation was transferred to a fixed stage of an intravital microscope based on an Olympus 
MVX10 Stereo Zoom platform (Center Valley, PA, USA) and equilibrated for 30 $\min$. During the 30 min period of equilibration, a sketch was made of the resistance network and respective vessel branches (FA, 1A, 2A, 3A) were identified for study, with the FA corresponding to the inferior gluteal artery. Images were acquired through a MV PLAPO 2XC objective (numerical aperture $=0.5$ ) coupled to a megapixel CCD camera (Stanford Photonics, Palo Alto, CA, USA) and displayed on a video monitor at $1000 \mathrm{X}$ final magnification. Digital images were recorded at 30 frames per second using Piper Controlled Imaging Desktop Software (Simi Valley, CA, USA). Internal diameters (IDs) were measured off-line as the width of the red blood cell column using video calipers using custom Labview software (National Instruments, Austin, TX) provided by Dr. Michael J. Davis (University of Missouri, Columbia, MO).

Diameters and vasomotor tone: Internal resting diameters (IDrest) of $F A, 1 A, 2 A$ and $3 \mathrm{~A}$ were measured at the end of the $30 \mathrm{~min}$ equilibration. Upon completion of the day's experiments, maximal IDs (ID $\max$ ) were measured for each branch during equilibration with sodium nitroprusside (SNP; $\left.10^{-4} \mathrm{M}\right)$ added to the superfusion solution to maximally dilate all branch orders $(48,111)$. Vasomotor tone of respective branch orders was quantified by calculating the difference between resting and maximal diameters compared to maximal diameter. The present experiments were performed in Control mice $(0 \mathrm{~d})$ and at 5, 10 and 21 of GM regeneration following $\mathrm{BaCl}_{2}$ injury; respective time points were studied in a randomized order. 
Reactivity to agonists: At each time point studied, after the initial $30 \mathrm{~min}$ equilibration period, endothelium-dependent vasodilation (EDD) was evaluated in response to the physiological agonist acetylcholine (ACh) (48) by cumulative addition ( $10^{-9} \mathrm{M}$ to $10^{-5} \mathrm{M}$ in $0.5 \mathrm{log}$ increments) to the superfusion solution. The IDs of respective vessel branch orders were recorded at each ACh concentration to generate concentration-response curves at each time point studied. The use of NE in preliminary experiments to evaluate $\alpha A R$ vasoconstriction resulted in vessel "sausaging" or "ballooning" where segments of vessel branches constricted while other segments dilated making it difficult to obtain a clear measure. Therefore, vasoconstriction to $\alpha_{1} A R$ stimulation with the pharmacological agonist phenylephrine (PE) was evaluated at the four defined time points during muscle regeneration by cumulative addition $\left(10^{-9} \mathrm{M}\right.$ to $10^{-5} \mathrm{M}$ in 0.5 log increments). The order in which PE and ACh evaluated was alternated across experiments; with the preparation superfused with control PSS for 30 min between respective agonists to restore vessels back to $I D_{\text {rest. }}$

Immunohistochemistry and imaging: For frozen sections, the GM muscle was processed as in (116) in the Cornelison laboratory. Following completion of intravital experiments, the GM tissue was excised and embedded in OCT (optical cutting temperature) freezing medium (Tissue Tek; Torrance, CA, USA) and frozen in isopentane cooled with liquid nitrogen. Specimens were then cryosectioned at $20 \mu \mathrm{m}$ thickness and blocked in $10 \%$ normal goat serum in phosphate-buffered 
saline (PBS) 1\% NP40 for 15 min. The primary antibody was then diluted in $10 \%$ goat serum in PBS (without detergent) and sections were incubated overnight at 4 ${ }^{\circ} \mathrm{C}$. Subsequently, samples were washed first with PBS and then with PBS containing $0.5 \%$ Tween20 (PBST). Concentrations of primary antibodies used were rabbit anti-laminin (Sigma) at 1:200 and mouse anti-eMyHC (DSHB) at 1:5. Samples were then incubated with secondary antibody in $10 \%$ goat serum in PBS for 3-5 hours, washed with PBS and PBST, fixed in 4\% PFA for 5-10 mins, and mounted in Vectashield. Images were acquired on an Olympus BX-61 microscope using Slidebook software (Intelligent Imaging Innovations), UPlanFL N objectives (0.50 NA), and a Retiga QImaging CCD camera (BC, Canada).

Data analysis and statistics: Spontaneous vasomotor tone was calculated as the difference between maximal and resting diameter normalized to maximal diameter $\left[\left(I D_{\max }-I D_{\text {rest }}\right) /\left(I D_{\max }\right)\right] \times 100 \%(48)$. Vasodilation and vasoconstriction were calculated as the change in ID from baseline at each respective agonist concentration. Data were analyzed with one- or two-way analysis of variance and post-hoc Bonferroni tests were conducted in cases of significant main effects. Summary data are presented as means \pm S.E. with $P<0.05$ accepted as statistically significant. 


\section{RESULTS}

Local delivery of $\mathrm{BaCl}_{2}$ injured $\mathrm{GM}$ myofibers and microvasculature. At $1 \mathrm{~d}$ post injury, injection of the fluorescent marker, FITC-dextran, revealed dark, unperfused regions of the GM indicative of microvascular disruption with loss of blood flow in the affected region (Figure 2.2). At this early time point, leakage of FITC-dextran $(70 \mathrm{kDa})$ from residual microvessels further illustrated the loss of microvascular structural integrity. At $5 d$ post injury, the presence of intravascular FITC-dextran in the lumen of microvessels confirmed that capillary perfusion was restored, and that dye leakage was minimal. Robust proliferation of capillaries was also apparent based upon FITC-dextran labeling.

\section{Histological analysis indicated regeneration and recovery of GM myofibers} after injury. The GM was sampled at $0 \mathrm{~d}$ (control) and at $5 \mathrm{~d}, 10 \mathrm{~d}$ and $21 \mathrm{~d}$ to define the time course of myofiber regeneration using histological markers. Myofiber borders were viewed by labeling for the basement membrane protein, laminin (green) and nuclei (blue) (Figure 2.3). For Control myofibers, diameter approximated $75 \mu \mathrm{m}$ with nuclei located at the periphery. Myofiber diameter was greatly reduced $5 d$ post injury, with centralized nuclei in a small fraction of myofibers. Myofiber diameter then increased at $10 \mathrm{~d}$ and by $21 \mathrm{~d}$ appeared similar to Control. Centrally located nuclei in $10 \mathrm{~d}$ and $21 \mathrm{~d}$ sections confirmed newly regenerated myofibers post injury. Myofibers in the early stages of regeneration were identified by staining with eMyHC (red), a developmental myosin isoform that 
is distinct from adult myosin isoforms. At $5 \mathrm{~d}$, clusters of small myofibers expressed eMyHC, while at 10d, the fading of eMyHC in some myofibers and disappearance of eMyHc in other myofibers with central nuclei indicated that the initial stages of regeneration were nearing completion. The absence of eMyHC at 21d in myofibers with central nuclei indicated that they had matured and expressed adult MHC.

\section{Elevated resting diameter and diminished vasomotor tone with muscle} injury. The GM was prepared for intravital experiments as described in Methods and four branch orders of the resistance network were studied (Figure 2.4). Resting diameter was significantly elevated $5 \mathrm{~d}$ post injury in all branch orders compared to Control. Although resting diameter began to decrease at $10 \mathrm{~d}$, it remained elevated in all branch orders compared to Control. At $21 \mathrm{~d}$ post injury, resting diameters were not different from Control (Figure 2.5A). Maximal diameter measured during equilibration with SNP $\left(10^{-4} \mathrm{M}\right)$ in the superfusion solution was not different between Control, $10 \mathrm{~d}$ and $21 \mathrm{~d}$ in all branch orders but was decreased in $1 \mathrm{~A}$ and $2 \mathrm{~A}$ at $5 \mathrm{~d}$ compared to Control (Figure 2.5B). Vasomotor tone was significantly impaired at $5 \mathrm{~d}$ in all branch orders compared to Control and remained so in FA and $1 \mathrm{~A}$ at $10 \mathrm{~d}$. Vasomotor tone improved between $5 \mathrm{~d}$ and $10 \mathrm{~d}$ post injury and recovered to control by $21 \mathrm{~d}$ in all branch orders (Figure 2.5C).

Endothelium-dependent vasodilation to $\mathrm{ACh}$. Endothelium-dependent vasodilation was evaluated in FAs and arterioles (1A, 2A and 3A) (Figure 2.4) using concentration-response curves to ACh (Figure 2.6). In Control networks, 
vasodilation increased in a sigmoidal, concentration-dependent manner for each branch order. During early regeneration at $5 d$, respective branch orders failed to respond to $\mathrm{ACh}$, thus vasodilation was significantly impaired compared to Control throughout the network. At 10d, ACh evoked only a slight dilation of FAs and the amount of dilation at this time point increased with branch order. By $21 \mathrm{~d}$, all branch orders dilated in a concentration-dependent manner to ACh and were not different from respective Control responses (Figure 2.6).

Adrenergic vasoconstriction to $\alpha_{1} A R$ stimulation. Vasoconstriction to $\alpha_{1} A R$ stimulation was evaluated in FAs and arterioles (1A, 2A and $3 \mathrm{~A}$ ) (Figure 2.4) using concentration-response curves to PE (Figure 2.7). In Control networks, vasoconstriction increased sigmoidally with $[P E]$. At $5 d$, vasoconstriction was significantly impaired in FAs and attenuated in arterioles compared to Control. At $10 d$, vasoconstriction was impaired in FAs but was greater than observed for Control in arterioles, which may be attributable to lower resting tone at this time point. By $21 \mathrm{~d}$, vasoconstriction recovered in all branch orders and was not different from Control.

\section{DISCUSSION}

Using a novel model of skeletal muscle injury in the mouse, this was the first study to evaluate when and to what extent the microvascular resistance network 
recovers during regeneration. In response to acute injury of the GM induced by local injection of $\mathrm{BaCl}_{2}$, microvascular perfusion was disrupted $1 \mathrm{~d}$ post injury and restored at $5 \mathrm{~d}$ with an abundance of capillary proliferation. Resting diameters of arterioles and their feed arteries were elevated through $5 d$ and $10 d$, as reflected in the loss of spontaneous vasomotor tone. By $21 \mathrm{~d}$ post injury, vasomotor tone was restored throughout the network, coincident with myofiber regeneration. Endothelium-dependent vasodilation and $\alpha$-adrenergic vasoconstriction were abolished coincident with myofiber injury and degeneration, were ineffective at $5 d$ post-injury and $21 \mathrm{~d}$ were not different from Control. These are the first data to define the time course for recovery of function in microvascular networks that regulate blood flow during regeneration of skeletal muscle following acute injury.

\section{Importance of vasomotor tone}

Resistance vessels in resting skeletal muscle maintain a partially constricted state known as basal vasomotor tone, reflecting spontaneous contraction of SMCs of the vessel wall in response to transmural pressure (68). An increase in transmural pressure results in vasoconstriction and a decrease in pressure results in vasodilation in accord with the myogenic response $(58,61)$. In resting muscle, spontaneous vasomotor tone establishes an operating point from which the resistance vasculature can either dilate to increase flow or constrict to reduce flow in response to vasoactive stimuli (68). In the present study, vasomotor tone was impaired throughout the network at early regeneration time-points, especially at $5 d$ post injury (Figure 2.5), suggesting SMCs were incapable of regulating blood flow 
during early stages of recovery from muscle injury. Thus, in association with myofiber injury, damage to SMCs of the microvessel wall explains the loss of vasomotor tone in my experiments.

The loss of blood flow with muscle injury results in tissue hypoxia $(61,91)$. Under hypoxic conditions, the release of ATP from red blood cells increases NO production in vascular ECs and vasodilation (70). With injury and inflammation, inducible NOS is expressed by macrophages that predominate in damaged and regenerating skeletal muscle to regulate and facilitate myogenic precursor cells in muscle healing (95). With activation of iNOS and the generation of NO required for new myofiber formation from muscle SCs $(3,95,117)$, it is likely that tissue levels of NO are elevated in the injured GM, which would promote vasodilation and loss of vasomotor tone. I tested for such an effect at $5 \mathrm{~d}$ using the NO synthase inhibitor L-NAME (1 mM, 30 min incubation). However, this treatment was without effect (data not shown), suggested that additional factors (e.g., SMC damage) contribute to the loss of vasomotor tone. Thus, future studies are needed to determine why vasomotor tone is impaired in resistance networks during the early stages of skeletal muscle injury.

\section{Endothelium-dependent vasodilation in regulating muscle blood flow}

The vascular endothelium plays an integral role in mediating vasodilation through the release of substances that affect the relaxation of vascular SMCs. Acetylcholine is a physiological agonist that, along with its analogues (e.g., 
carbachol), has been used widely to evaluate endothelium-dependent relaxation of vascular SMCs $(10,21,44,48,51)$. The binding of ACh to muscarinic (M3) receptors of ECs stimulates phospholipase $\mathrm{C}$ to liberate inositol triphosphate $\left(\mathrm{IP}_{3}\right)$, increase $\left[\mathrm{Ca}^{2+}\right]_{i}$ and generate NO by activating eNOS. This gaseous signaling molecule and vasodilator, NO, hyperpolarize SMCs and cause relaxation by activating soluble guanylyl cyclase and increasing cGMP leading to PKG phosphorylation and activation of calcium-dependent potassium channels and the stimulation of $\mathrm{Na}^{+} / \mathrm{K}^{+}$ATPase activity in SMC membranes $(4,34,54,68)$. Because inhibiting NO and other endothelium-dependent vasodilators such as prostacyclin $\left(\mathrm{PGI}_{2}\right)$ can only partially attenuate vasodilation, particularly in the resistance vasculature, an alternative signaling pathway entails endothelium dependent hyperpolarization $(E D H)$ of SMCs to cause their relaxation $(15,34,52,68)$. In such manner, an increase in intracellular $\mathrm{Ca}^{2+}$ concentration within ECs activates small and intermediate $\mathrm{Ca}^{2+}$-activated $\mathrm{K}^{+}$channels, with efflux of $\mathrm{K}^{+}$resulting in $\mathrm{EC}$ hyperpolarization $(15,25,52)$. In turn, hyperpolarization spreads along the endothelium and into surrounding SMCs via myoendothelial gap junctions (15, 34, $44,52)$.

With impaired spontaneous vasomotor tone (Figure 2.5) and negligible vasodilation to $\mathrm{ACh}$ at $5 \mathrm{~d}$ post injury in all branch orders and in proximal branches (FA and $1 A$ ) at 10d (Figure 2.6), further studies are needed in determining whether impaired vasodilation is due to damaged signaling in ECs or to the response of SMCs to signals originating in ECs upon administration of ACh. Since the 
inflammatory response to muscle injury contributes to elevate NO production as discussed above $(3,95,117)$, it may be expected that stimulation with ACh would not cause additional vasodilation. Further studies are also needed in determining to what extent $\mathrm{BaCl}_{2}$ injury in the $\mathrm{GM}$ results in damage to both the $\mathrm{EC}$ and $\mathrm{SMC}$ layers of the microvessel wall resulting in impaired endothelium-dependent vasodilation. There are no previous studies that have evaluated endotheliumdependent vasodilation during regeneration after skeletal muscle injury. My experiments are the first to show loss of endothelium-dependent vasodilation coincident with myofiber degeneration, which then gradually recovers to Control by $21 \mathrm{~d}$ post injury, providing a timeline for recovery of vasodilatory mechanisms that contribute to blood flow regulation in skeletal muscle.

\section{Adrenergic vasoconstriction in regulating muscle blood flow}

Although multiple membrane receptors and ion channels are involved in SMC contraction through several signaling pathways, I focused on vasoconstriction regulated by vascular ARs because of their role in regulating arterial blood pressure through the release of NE from perivascular sympathetic nerve terminals $(68,80)$. The binding of $\mathrm{NE}$ to $\alpha_{1} \mathrm{AR}$ and $\alpha_{2} \mathrm{AR}$ increases $\left[\mathrm{Ca}^{2+}\right]_{\mathrm{i}}$ in response to $\mathrm{Ca}^{2+}$ influx through the cell membrane or its internal release from the sarcoplasmic reticulum, resulting in vasoconstriction $(68,115)$. Due to difficulties in measuring vasoconstriction with NE (vessels exhibited "sausaging" with segments of constriction alternated with segments of dilation; not shown), my studies specifically focused on evaluating recovery of vasoconstriction to $\alpha_{1} A R$ stimulation 
with $\mathrm{PE}$, where uniform vasoconstriction of a segment is achieved from liberation of $\mathrm{IP}_{3}$ via phospholipase $\mathrm{C}$ activity and increases in $\left[\mathrm{Ca}^{2+}\right]_{\mathrm{i}}(43)$. As the functional distribution of $\alpha_{1} A R$ subtype varies with branch order in resistance networks of skeletal muscle (80), I investigated sympathetic vasoconstriction in four successive branch orders of the resistance network to determine when and to what extent vasoconstriction via $\alpha_{1} A R$ stimulation recover during regeneration. My data suggest that due to muscle injury, sympathetic vasoconstriction in response to $\alpha_{1}$ receptor stimulation is impaired in all branch orders studied during the early stages of regeneration (e.g. 5d) (Figure 2.7).

With local injury induced by $\mathrm{BaCl}_{2}$, tissue damage likely encompasses perivascular sympathetic nerves as well as SMCs. Thus, adrenergic vasoconstriction may be expected to be impaired during regeneration. However, by $10 \mathrm{~d}$ post injury sympathetic vasoconstriction was restored indicating that, with completion of initial phase of muscle regeneration (Figure 2.3), vasoconstriction in response to $\alpha_{1} A R$ stimulation has also recovered. By $21 \mathrm{~d}$ post injury, $\alpha_{1} A R$-mediated vasoconstriction was not different from Control (Figure 2.7), confirming restoration of this signaling pathway. An early study evaluating vasoconstriction in the presence of NE ( $\alpha_{1} A R$ and $\alpha_{2} A R$ agonist) in hamster cheek pouch skeletal muscle grafts reported diminished responses to NE that recovered over 3-4 months after grafting, and concluded that impaired vasoconstriction was due to discontinuity in the SMC layer or from irregularities in its organization (24). Further studies are 
needed in evaluating structural damage to sympathetic nerve terminals and SMC aARs to explain functional data acquired through my experiments.

\section{SUMMARY AND CONCLUSION}

Using an in vivo mouse model in evaluating vascular reactivity and blood flow regulation, the present study provides new insight into when and to what extent microvascular resistance network function recovers during regeneration following injury to skeletal muscle. Spontaneous vasomotor tone in resting muscle allows the resistance vasculature to either dilate or constrict in response to vasoactive stimuli and to thereby regulate muscle blood flow. The diminished vasomotor tone observed at early stages of regeneration suggests that muscle blood flow regulation is impaired with skeletal muscle injury. The elevated resting diameter observed in all branch orders studied may be integral to maximizing blood flow to deliver oxygen and nutrients needed for myofiber regeneration as well for the removal of cellular debris and byproducts from damaged tissue. Although impaired at $5 d$ during early regeneration, vasomotor tone improved when initial stages of myofiber restoration neared completion at $10 \mathrm{~d}$ and had recovered by $21 \mathrm{~d}$ when myofibers matured as shown in my histological studies. These findings support my hypothesis that restoration of flow control coincides with maturation and recovery of myofibers. Similar to recovery of vasomotor tone, EDD improved at $10 \mathrm{~d}$ and recovered by $21 \mathrm{~d}$ suggesting that recovery of vasomotor tone is requisite to the manifestation of EDD. Gradual recovery of EDD also suggests recovery of EC function and vasodilatory mechanisms, especially in distal arterioles recovering 
sooner than proximal vessels aiding in regulating the distribution of blood flow within the muscle. Although impaired at $5 \mathrm{~d}$, the ability to constrict at $10 \mathrm{~d}$ with PE suggests that $S M C$ responses to $\alpha_{1} A R$ stimulation is restored. With vasomotor tone recovered by $21 \mathrm{~d}$ and vasoconstriction to PE not different from Control in the branch orders studies, it can be stated that SMC function is restored by $21 \mathrm{~d}$. Future studies are required to understand mechanisms of impaired vasomotor tone, EDD and vasoconstriction with injury. Such insight will contribute towards developing appropriate therapeutic interventions to facilitate recovery of blood flow regulation in accord with the metabolic demands of skeletal muscle recovering from injury. 


\section{FIGURES}

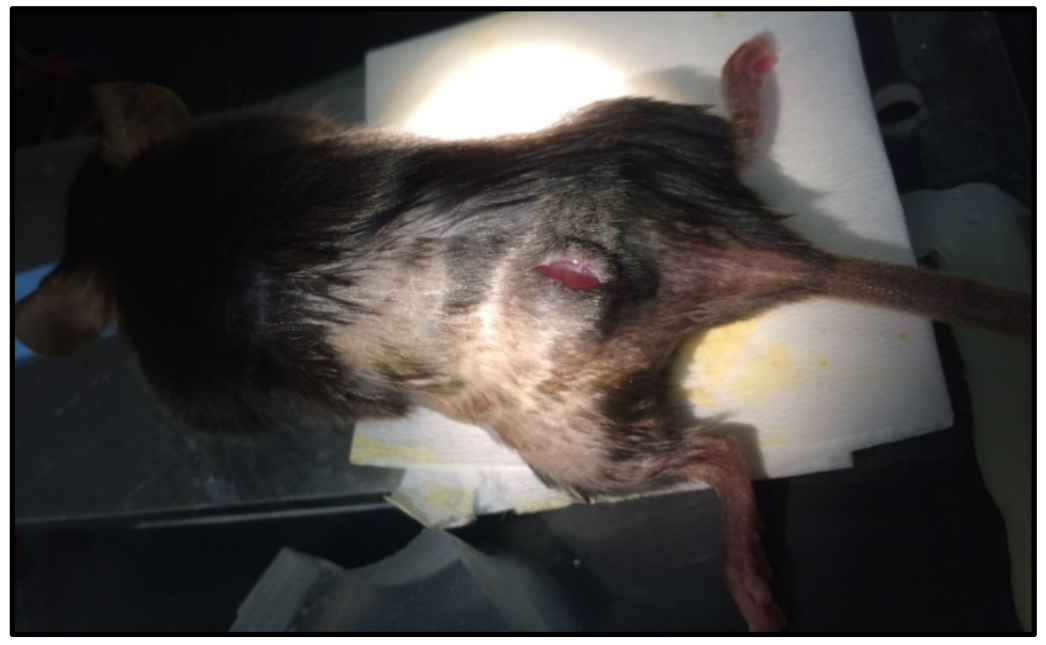

Figure 2. 1. $\mathrm{BaCl}_{2}$ injury of mouse $\mathrm{GM}$.

A $\sim 5 \mathrm{~mm}$ incision was made through the skin overlying the $\mathrm{GM}$ and $75 \mu \mathrm{l}$ of $1.2 \% \mathrm{BaCl}_{2}$ solution was injected into the space between the GM and gluteus medius muscles to damage GM myofibers and its microvasculature. 
Control

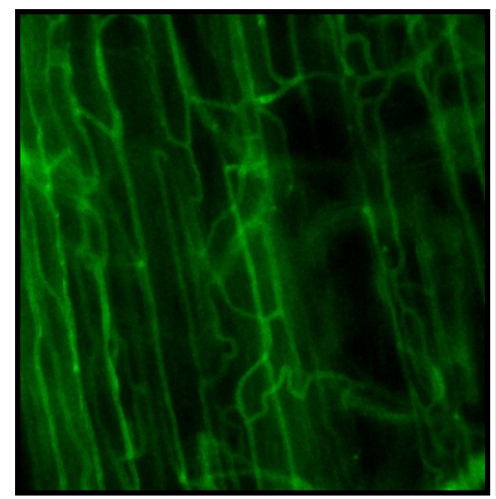

$1 d$

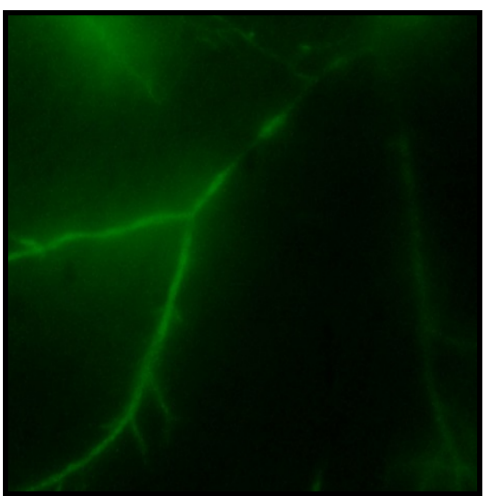

$5 d$

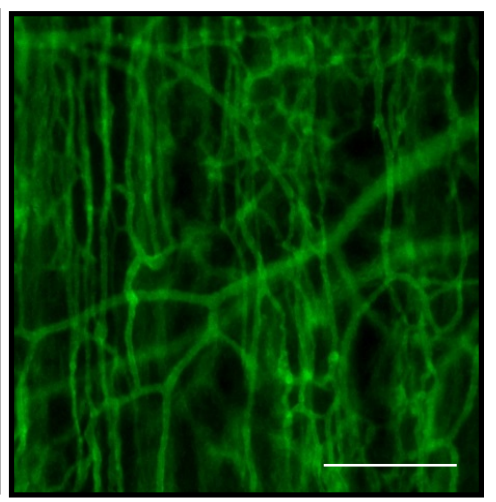

Figure 2. 2. In vivo fluorescence imaging showing microvascular damage with $\mathbf{B a C l}_{2}$.

FITC-dextran $(70 \mathrm{kDa}$ ) labeling (via a retro-orbital injection) of the microvascular network in control (left), loss of capillary perfusion with disruption of the microvasculature at $1 \mathrm{~d}$ following administration of $\mathrm{BaCl}_{2}$ (middle) and recovery of capillary perfusion at $5 \mathrm{~d}$ (right) post injury. Note greater number and tortuousity of capillaries along regenerating myofibers vs Control. Scale bar $=$ $100 \mu \mathrm{m}$ and applies to all panels. 
Control

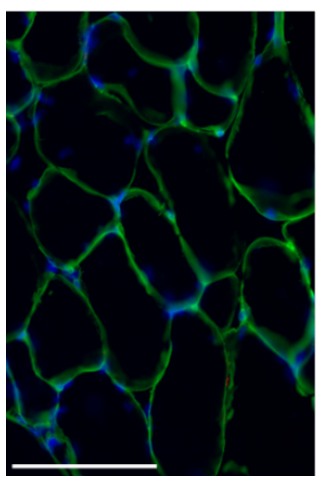

$5 d$

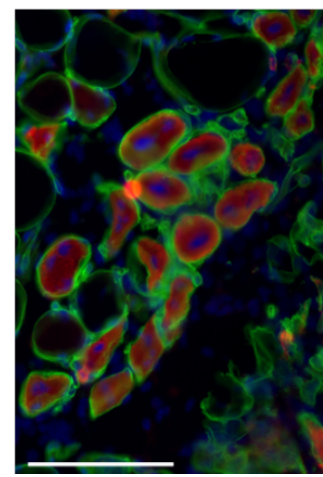

10d

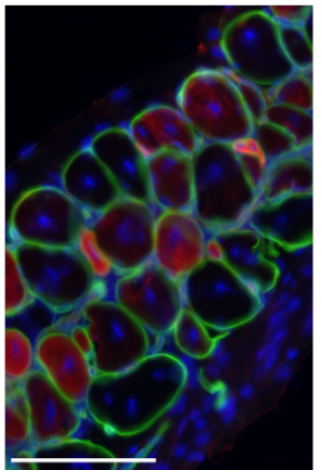

21d

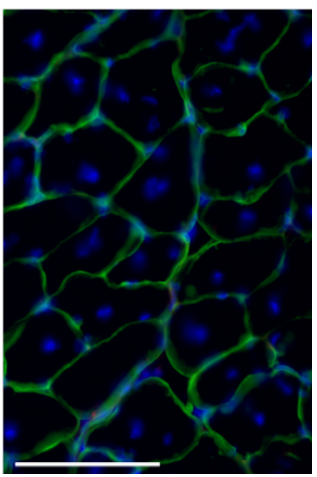

Figure 2. 3. Histology of regenerating skeletal muscle.

Cross sections of GM immunostained for the extracellular matrix protein laminin (green) and embryonic myosin heavy chain (eMHC, red) to identify regenerating muscle fibers at $5 \mathrm{~d}$ and $10 \mathrm{~d}$ post injury compared to Control (0d). Note central location of nuclei (blue, DAPI) in regenerating fibers and loss of eMHC by 21d with persistence of central nuclei. Scale bars $=100 \mu \mathrm{m}$ (Credits: Rylie White, Cornelison laboratory) 

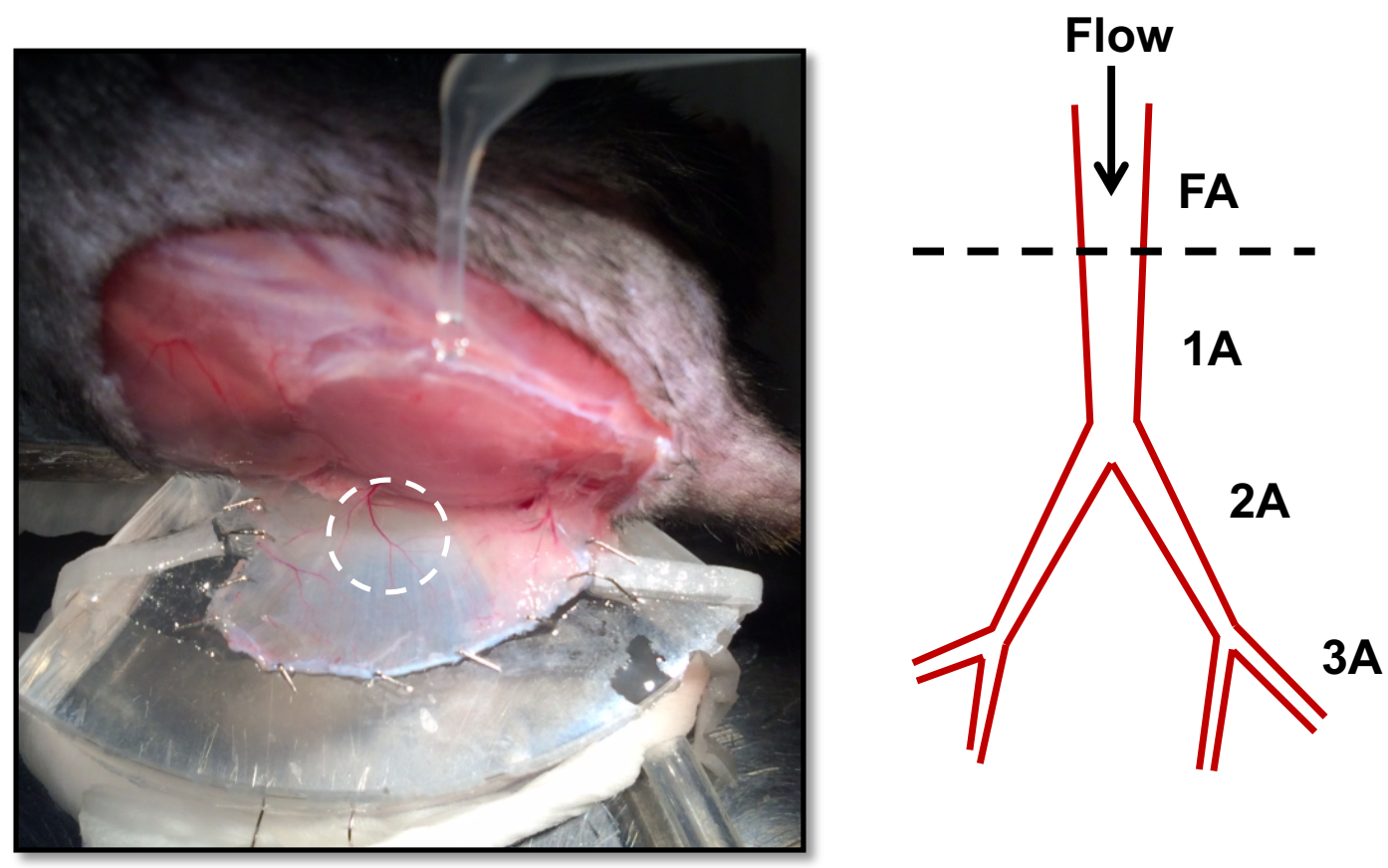

Figure 2. 4. GM muscle preparation and branch orders of study.

The GM is dissected free along lumbar vertebrae and iliac crest, reflected away from the mouse, pinned onto a transparent Sylgard pedestal and irrigated with PSS at $35^{\circ} \mathrm{C}$ (left). Schematic corresponding to region within broken circle showing inferior gluteal artery external to the $G M$, defined as the feed artery (FA), which enters the muscle (horizontal broken line) and becomes a first-order (1A) arteriole, which gives rise to second-order (2A) and then third-order (3A) arterioles (remaining network branches not depicted for clarity). 
$\square$ Control $\square$ 5d $\square$ 10d $\square$ 21d

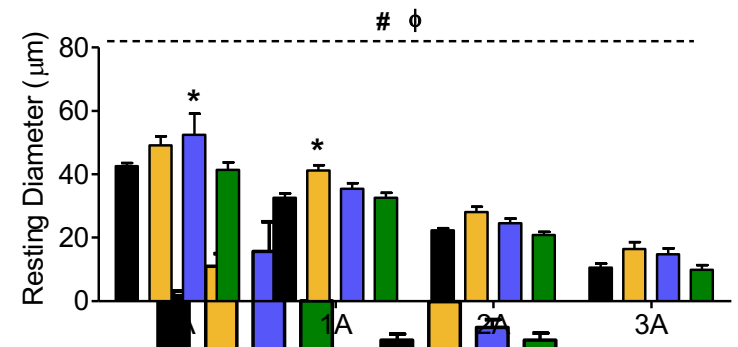

Figure 2. 5. Resting diameters, maximal diameters and vasomotor tone.

(A) Resting internal diameters were increased at $5 \mathrm{~d}$ and $10 \mathrm{~d}$ and recovered by 21d post injury. (B) Maximal diameters during topical sodium nitroprusside $\left(10^{-4} \mathrm{M}\right)$ were maintained throughout recovery except for a transient reduction in arterioles at $5 \mathrm{~d}$.

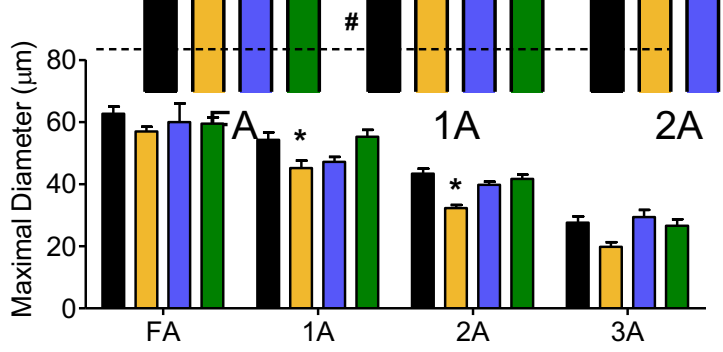

(C) Vasomotor tone was decreased at $5 \mathrm{~d}$ and $10 \mathrm{~d}$ post injury and recovered by $21 \mathrm{~d}$. Summary data are means \pm $\mathrm{SE} ; \mathrm{n}=5$ /group. \# $\mathrm{P}<0.05$, main effect of time. $\Phi P<0.05$, main effect of branch order. ${ }^{*} \mathrm{P}<0.05$ vs. Control

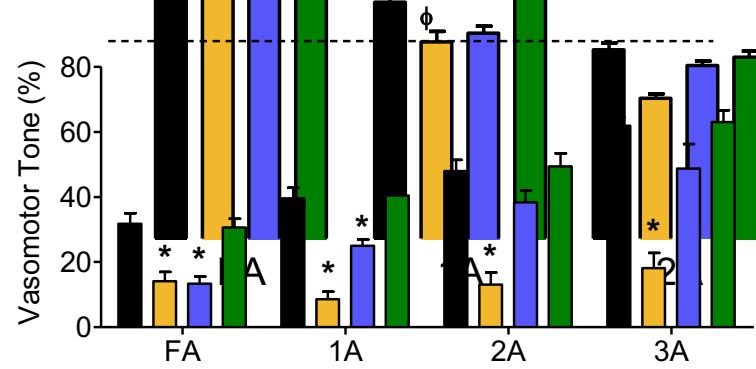




\section{$\square$ Control $\square$ 5d $\square$ 10d $\square$ 21d}

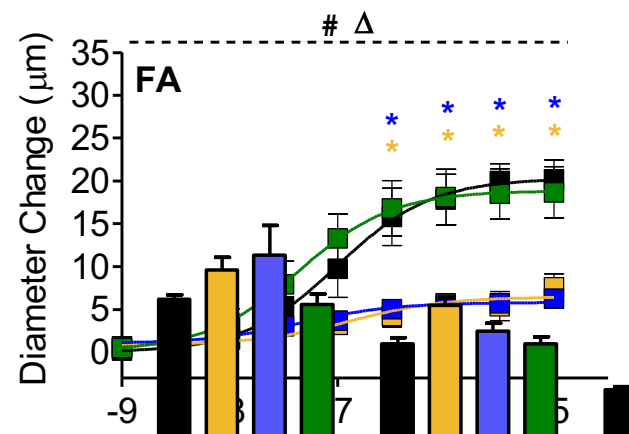

Figure 2. 6. Concentration-response curves to ACh.

Endothelium-dependent vasodilation was depressed at $5 \mathrm{~d}$ in all branch orders. At 10d, vasodilation was depressed in FA and $1 \mathrm{~A}$ but improved with increasing branch order. At 21d, vasodilation was not different from Control for all branch orders. Summary data are means $\pm S E ; n=5$ per group. $\# P<0.05$, main effect of time. $\triangle P<0.05$,

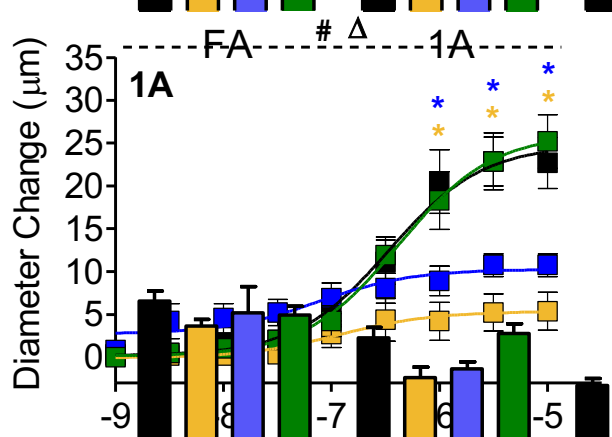
main effect of $[\mathrm{ACh}] .{ }^{*} \mathrm{P}<0.05$ vs. Control
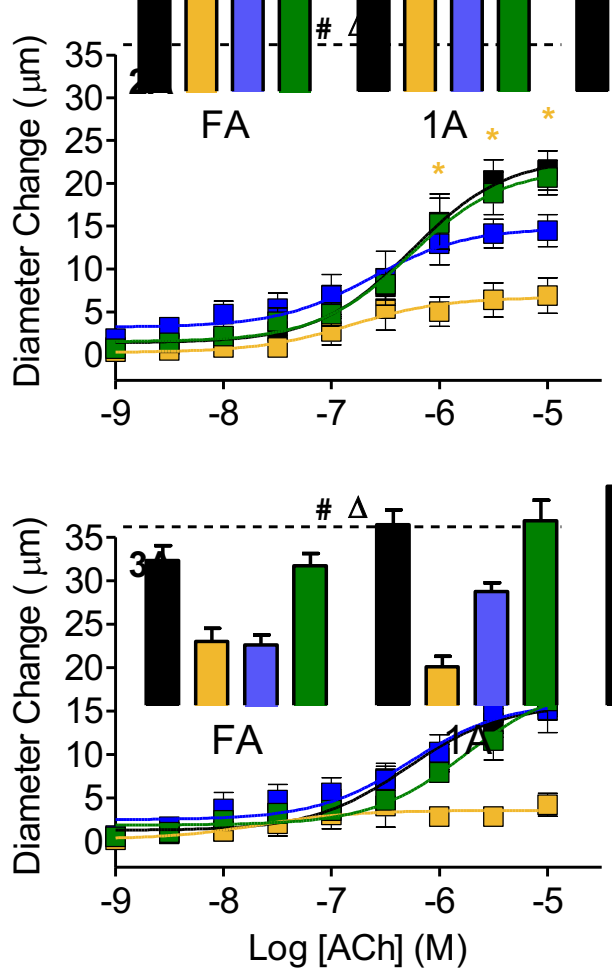

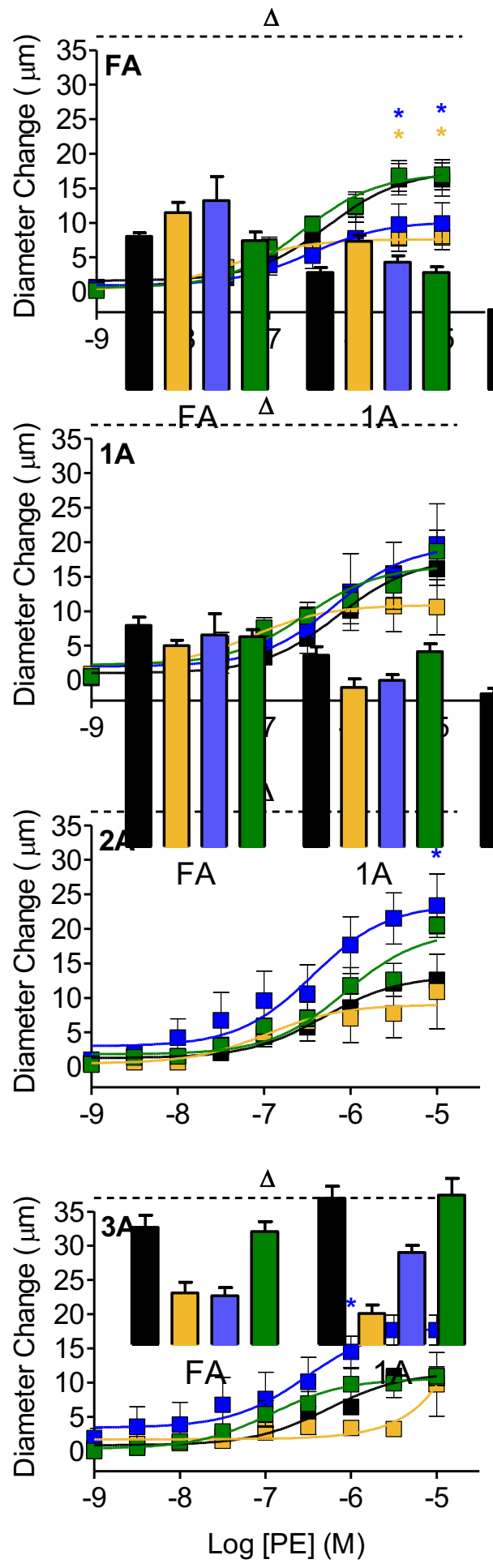

Figure 2. 7. Concentration-response curves to PE.

Vasoconstriction to $\alpha_{1} A R$ stimulation decreased at $5 \mathrm{~d}$ vs. Control in all branch orders. At $10 \mathrm{~d}$, vasoconstriction was decreased in FA but increased with branch order. By 21d, vasoconstriction was not different from control in all branch orders. Summary data are means $\pm S E ; n=5$ per group. $\# P<0.05$, effect of time. $\triangle P<0.05$, effect of [PE]. * $\mathrm{P}<0.05$ vs Control. 


\section{CHAPTER 3}

\section{RECOVERY OF FUNCTIONAL VASODILATION DURING SKELETAL MUSCLE REGENERATION}

\section{INTRODUCTION}

Functional vasodilation entails the increase in blood flow to contracting skeletal muscle in accord with the intensity and duration of physical activity. A hallmark of musculoskeletal health is the ability to quickly match muscle blood flow to metabolic demand $(63,68,81,86,126)$. Skeletal muscle injuries result from overexertion and physical trauma, exposure to myotoxic agents (e.g. venomous reptile bites) and ischemia, with the majority of injuries related to strains, contusions and lacerations $(42,65,67,92)$. Fortunately, skeletal muscle has resident muscle-specific stem cells known as satellite cells, that are activated, proliferate and differentiate to enable regeneration of myofibers $(29,39,45,67$, 83), Although many studies have investigated myofiber regeneration, little is known regarding the recovery of functional vasodilation. The microvasculature within skeletal muscle is damaged with muscle injury, therefore understanding when and to what extent functional vasodilation recovers during muscle regeneration is integral to supplying the oxygen and nutrients needed for muscle repair, removal 
of cellular debris and metabolic by-products, and restoring the ability to engage in physical activity.

Muscle blood flow increases rapidly with the onset of exercise and plateaus at a steady-state that is proportional to the level of contractile activity $(32,84,109,123)$, which under experimental conditions (e.g., electrical stimulation) is controlled through changing stimulation frequency and the duration of contraction $(63,81$, 125). A key determinant of muscle force production is motor unit recruitment via the firing of alpha motor neurons in the spinal cord, and vasodilation increases in direct proportion to motor unit recruitment (125). The rapid onset vasodilation (ROV) observed upon initiating exercise can be evoked by a single tetanic contraction, whereas slow onset (i.e., steady-state) vasodilation (SOV) observed during sustained activity can be evoked by rhythmic twitch contractions (63). Thus, varying stimulation parameters enables evaluation of respective mechanisms of functional vasodilation and the ensuing increases in muscle blood flow. Previous studies have evaluated recovery of skeletal muscle force generation after injury $(22,96,108,127)$, however there are no studies of the coupling between active force production and functional vasodilation during skeletal muscle regeneration in vivo, particularly with respect to microvessels that actually control muscle blood flow.

The gluteus maximus muscle $(\mathrm{GM})$ is common to both sexes, found in all mammalian species and, as a powerful extensor of the hip, is recruited during 
locomotion. In the mouse, the thinness of the GM and its superficial location make it an ideal muscle to study mechanisms of functional vasodilation in vivo using intravital microscopy $(48,111)$. With respect to the control of muscle blood flow, feed arteries (FA) which are located external to the muscle enter the tissue and become first order arterioles $(1 \mathrm{~A})$, which branch into second order arterioles $(2 \mathrm{~A})$, which branch into third order arterioles (3A). Proximal arterioles (FA and $1 \mathrm{~A}$ ) regulate the amount of blood flow entering the muscle whereas distal arterioles (2A and $3 A$ ) regulate the distribution of blood flow within the muscle (104). As respective branches of the resistance network are readily observed in the GM prepared for intravital imaging, it provides the opportunity to study their response to muscle injury with reference to the regeneration of myofibers. To injure the GM and its microvasculature, I used local injection of barium chloride $\left(\mathrm{BaCl}_{2}\right)$, which is myotoxic yet preserves muscle satellite cells, thereby enabling myofiber regeneration to occur following injury $(56,96)$. I studied defined branches of the microvascular resistance network at key time points defined in my preliminary studies. Using electrical field stimulation, I evaluated the recovery of ROV and SOV by manipulating the frequency and duration of muscle contraction. To gain insight into corresponding changes in skeletal muscle function, I evaluated the frequency force relationship at respective time-points to test the hypothesis muscle active force and functional vasodilation recover in parallel during skeletal muscle regeneration. 


\section{METHODS}

Barium Chloride injury and time points of study: All animal procedures were conducted according to protocols approved by the Animal care and Use Committee of the University of Missouri, Columbia. Male C57BL/6J mice were obtained from Jackson Laboratory (Bar Harbor, ME, USA) at $~ 3.5$ months of age and acclimated at the University of Missouri animal care facilities at least 1 week prior to study. For barium chloride $\left(\mathrm{BaCl}_{2}\right)$ injury, a mouse was anesthetized with ketamine/xylazine (100/10 mg/kg; intraperitoneal injection) and hair was shaved from the skin overlying the GM. The area was sterilized with Betadine Solution (Purdue Products L.P. Stamford, CT, USA), wiped with $70 \%$ alcohol, and then a 5 $\mathrm{mm}$ incision was made through the skin. With this access, $75 \mu \mathrm{l}$ of $1.2 \%(\mathrm{v} / \mathrm{v})$ of $\mathrm{BaCl}_{2}$ (LabChem, Zelienople, PA, USA) solution was injected using a Hamilton syringe and needle (32 gauge, $0.5^{\prime \prime}$ long, $30^{\circ}$ tip bevel; Reno, NV, USA) into the space between the GM and underlying gluteus medius muscle to damage myofibers and microvascular networks of the GM. The incision was closed with VetClose surgical glue (Henry Schein, Dublin, OH, USA) and monitored closely until animals regained consciousness and for 4 consecutive days post injury to ensure healing of the incision and resumption of normal activity. The microcirculation of the GM was studied at 5, 10, 21 and 35d post injury with reference to uninjured Control $(0 \mathrm{~d})$. 
Intravital microscopy: The GM was prepared for intravital microscopy as described $(48,80,111)$. On the day of an experiment, a mouse was anesthetized using an intraperitoneal injection of pentobarbital sodium $(60 \mathrm{mg} / \mathrm{kg})$, dorsal skin over the left GM was shaved and the mouse was placed on a warming plate in the prone position to maintain body temperature $\left(37^{\circ} \mathrm{C}\right)$ throughout experiments. While viewing through a stereomicroscope, the skin and connective tissue overlaying the GM were removed while the exposed tissue was continuously superfused at $\sim 3$ $\mathrm{mL} / \mathrm{min}$ with a bicarbonate-buffered physiological salt solution (PSS; $34^{\circ} \mathrm{C}, \mathrm{pH} 7.4$ ) containing (in mM) $131.9 \mathrm{NaCl}, 4.7 \mathrm{KCl}, 2 \mathrm{CaCl}_{2}, 1.17 \mathrm{MgSO}_{4}$, and 18 $\mathrm{NaHCO}_{3}$ equilibrated with $5 \% \mathrm{CO}_{2} / 95 \% \mathrm{~N}_{2}$. The $\mathrm{GM}$ was dissected along its insertion (lumbar vertebra and iliac crest) and secured at the edges with stainless steel pins (diameter: $0.2 \mathrm{~mm}$ ) onto a transparent rubber pedestal (Sylgard 184; Dow Corning, Midland, MI, USA) to approximate in situ dimensions (48). The completed preparation was transferred to the stage of an intravital microscope based on an Olympus MVX10 Stereo Zoom platform (Center Valley, PA, USA) and equilibrated for $30 \mathrm{~min}$. Images were acquired through a MV PLAPO 2XC objective (numerical aperture $=0.5$ ) coupled to a megapixel CCD camera (Stanford Photonics, Palo Alto, CA, USA) and displayed on a video monitor at 1000X final magnification. Digital images were recorded at 30 frames per second using Piper Controlled Imaging Desktop Software (Simi Valley, CA, USA) and internal diameters (IDs) were measured using video calipers. Images were analyzed using custom Labview software (National Instruments, Austin, TX, USA) provided by Dr. Michael J. Davis (University of Missouri, Columbia, MO). Supplemental doses (20 
$\mathrm{mg} / \mathrm{kg}$ ) of pentobarbital sodium were given (intraperitoneal injection) to maintain a stable plane of anesthesia throughout experiments and the mouse was euthanized at the end of experiments with an overdose of pentobarbital sodium followed by cervical dislocation.

Diameters and vasomotor tone: During the $30 \mathrm{~min}$ period of equilibration, a sketch was made of the resistance network and respective vessel branches were identified for study, with the FA corresponding to the inferior gluteal artery. Resting diameters (IDrest) of FA, $1 \mathrm{~A}, 2 \mathrm{~A}$ and $3 \mathrm{~A}$ were measured at the end of the $30 \mathrm{~min}$ equilibration. Upon completion of the day's experiments, maximal IDs (ID $\max )$ were measured for each branch during equilibration with sodium nitroprusside (SNP; $10^{-}$ ${ }^{4} \mathrm{M}$ ) added to the superfusion solution to maximally dilate all branch orders (48, 111). Vasomotor tone of respective branch orders was measured by calculating the difference between resting and maximal diameters normalized to maximal diameter.

Contraction protocol: Contraction of the GM was evoked with electrical field stimulation using platinum-iridium (90\%-10\%) electrodes (diameter: $250 \mu \mathrm{m}$ ) positioned on each side of the muscle. Monophasic pulses $(8 \mathrm{~V}, 0.1 \mathrm{~ms})$ were delivered from an S48 stimulator coupled to a SIU5 stimulation isolation unit (Grass Instruments West Warwick, RI, USA). Tetanic contractions for ROV and rhythmic twitch contractions for SOV were randomized across experiments, however contraction duration (100, 250 and $500 \mathrm{~ms}$ for ROV) and contraction frequency ( 2 
$\mathrm{Hz}$ and $4 \mathrm{~Hz}$ for SOV) were always applied in increasing order. Separate stimuli were given for each vessel branch and the sequence in which respective branch orders was also randomized across experiments. Active force measurements required clamping of the muscle, which compressed microvessels within the clamped region. Therefore, vasomotor responses to muscle contraction and active force measurements were evaluated in separate experiments.

Tetanic contractions and rapid onset vasodilation: A single brief tetanic contraction was elicited at $100 \mathrm{~Hz}(48,63,86)$ which elicits maximal tetanic force. Individual contractions were 100, 250 and $500 \mathrm{~ms}$ in duration with IDs recorded at rest and in response to contraction. Resting and peak response IDs were measured off line from recordings of respective contraction durations. Vessels returned to their resting baseline diameters within a few seconds after each contraction and allowed a $2-3$ min recovery period before eliciting the next contraction.

Rhythmic contractions and slow onset vasodilation: Slow onset vasodilation was measured by contracting the $\mathrm{GM}$ at $2 \mathrm{~Hz}$ and $4 \mathrm{~Hz}$, each for $30 \mathrm{~s}$, and peak ID was measured at $30 \mathrm{~s}$ after reaching a plateau at $15-20 \mathrm{~s}$. Stimulation at these frequencies generated unfused twitch contractions. Following each $30 \mathrm{~s}$ period contraction, all vessel branch orders returned to baseline within $4-5$ min.

Active force production: In separate experiments, the GM was prepared for active force measurements $(14,48)$. While irrigated continuously with PSS at 34- 
$35^{\circ} \mathrm{C}$, the $\mathrm{GM}$ was dissected as described above, clamped across its entire width, perpendicular to myofiber orientation, using an atraumatic clamp. The clamp was secured to a load beam (LCL-1136; Omega, Stamford, CT, USA) coupled to a Transbridge amplifier (TBM-4; World Precision Instruments, Sarasota, FL, USA). The load beam was attached to a micrometer that enabled muscle length to be adjusted to elicit peak twitch tension $\left(L_{o}\right)$, determined for each experiment by adjusting the length of the muscle during twitch contractions $(1 \mathrm{~Hz}, 20 \mathrm{~V})(48)$. To define the frequency-force relationship, the GM was stimulated at $10-120 \mathrm{~Hz}$ in $20 \mathrm{~Hz}$ increments (each for $1 \mathrm{~s}$ ) with peak force reached within $200 \mathrm{~ms}$. Maximal active force (g) was measured by calculating the difference between peak force for each contraction and the preceding resting force. Following force measurements, the GM was severed along the edge of the clamp and cut from its origin, blotted of excess moisture and its wet weight $\left(\mathrm{W}_{\mathrm{w}}\right)$ was measured $( \pm 0.01$ mg) on a microbalance (XS105; Mettler Toledo, Columbia, OH, USA). Muscle cross-sectional area $(\mathrm{CSA})\left(\mathrm{mm}^{2}\right)$ was calculated as: $\mathrm{W}_{\mathrm{w}} /\left(\mathrm{L}_{\circ} \cdot\right.$ muscle density) with muscle density $=1.06 \mathrm{mg} \cdot \mathrm{mm}^{-3}(106)$. Active force produced by the $\mathrm{GM}$ was plotted as a function of stimulation frequency for values measured in $\mathrm{g}$ and after normalization to muscle mass or CSA $\left(\mathrm{mm}^{2}\right)$.

Histological assessment with hematoxylin and eosin: At the end of an experiment, the excised GM was fixed in $10 \%$ formalin. Muscles were embedded in paraffin and $5 \mu$ m-thick sections were stained with hematoxylin and eosin (IDEXX, Columbia, MO, USA). Sections were imaged using Köhler illumination 
with a 20X objective (NA, 0.5) on an E800 microscope using NIS Elements Advanced Research software (Nikon, Tokyo, Japan).

Data analysis and statistics: For each vessel branch, spontaneous vasomotor tone was calculated as the difference between maximal and resting diameter normalized to maximal diameter $\left[\left(I D_{\max }-I D_{\text {rest }}\right) /\left(I D_{\max }\right)\right] X 100 \%(48,111)$. Vasodilation was expressed as the actual change in diameter recorded for each vessel branch: (IDresp - ID rest), where ID resp is the peak (ROV) or plateau (SOV) ID for a given contraction and $I D_{\text {rest }}$ is the preceding resting ID. To compare relative responses across time points and vessel branch orders, respective changes in diameter were calculated as the difference between ID resp and ID rest normalized to $I D_{\text {max }}:\left[\left(I D_{\text {resp }}-I D_{\text {rest }}\right) / I D_{\max }\right] X 100 \%$. To measure temporal aspects of ROV (at 500 ms contraction duration in FA, $1 A, 2 A$ and $3 A$ branch orders at $5 d, 10 d, 21 d, 35 d$ post injury and Control) change in diameter was measured every s for the first 11 $s$ and thereafter every two $s$ ( 2 data points) and then five $s$ (1 data point) post contraction. Diameter at time point $0 \mathrm{~s}$ was measured at the cessation of contraction due to tissue displacement during contraction. Data were analyzed with one- or two-way analysis of variance (GraphPad Prism 5; GraphPad, La Jolla, CA, USA), and when F-ratios were significant, post-hoc Bonferroni post-hoc tests were performed. Summary data are presented as means \pm S.E. with $P<0.05$ accepted as statistically significant. 


\section{RESULTS}

Regenerating myofibers have centralized nuclei. As shown with hematoxylin and eosin staining, damaged myofibers were observed at $5 \mathrm{~d}$ along with nuclei of (presumably) inflammatory cells, confirming $\mathrm{BaCl}_{2}$ injury to the $\mathrm{GM}$. This pathological state precluded evaluation of active force or functional vasodilation at 5d. At 10d, new myofibers were present throughout the GM cross-section, characterized by smaller diameter and centralized nuclei. Myofiber diameter increased at $21 \mathrm{~d}$ compared to $10 \mathrm{~d}$, consistent with maturation of nascent myofibers. By $35 \mathrm{~d}$, a subpopulation of myofibers with central nuclei appeared larger in diameter when compared to Control (Figure 3.1).

\section{Recovery of resting diameter and vasomotor tone during myofiber} regeneration. Resting diameters were significantly greater than Control at $5 d$ in all branch orders. Although beginning to recover at $10 \mathrm{~d}$, resting diameters remained greater than Control. At 21d, resting diameters were no longer different from Control and were maintained at 35d (Figure 3.2 - A). With 2 exceptions (2A and $3 A$ at $5 d$ ), maximal diameters were not different between time-points across branch orders (Figure 3.2 - B). Spontaneous vasomotor tone was significantly impaired in all branch orders at $5 \mathrm{~d}$ post injury compared to control and although improved from 5d, was still significantly impaired at 10d. Vasomotor tone recovered by $21 \mathrm{~d}$, maintained at $35 \mathrm{~d}$ and was not different from Control at these later time points (Figure $3.2-\mathrm{C}$ ). 
Recovery of rapid onset vasodilation (ROV) during myofiber regeneration. Compared to Control, the peak response diameter during ROV was impaired at $5 \mathrm{~d}$ in FA, 1A, 2A and $3 \mathrm{~A}$ branch orders at all contraction durations $(100,250$ and 500 ms) evaluated (Figure 3.3). Although improved relative to 5d, ROV was still impaired at $10 \mathrm{~d}$. Peak responses during ROV improved further at $21 \mathrm{~d}$ and recovered to approximate control by $35 \mathrm{~d}$ in all branch orders at all contraction durations. (Figure 3.3 and Figure 3.4). Relative responses increased with branch order and over time within each branch order. The time course of ROV at $500 \mathrm{~ms}$ contraction duration, particularly the time-to-peak response, appeared delayed compared to Control at $5 \mathrm{~d}$ and became progressively more rapid at $10 \mathrm{~d}, 21 \mathrm{~d}$ and 35d (Figure 3.5).

Recovery of slow onset vasodilation (SOV) during myofiber regeneration. Compared to Control, the peak response during SOV was also impaired at $5 \mathrm{~d}$ in all branch orders at both contraction frequencies (2 and $4 \mathrm{~Hz}$ ). SOV improved at $10 \mathrm{~d}$ when compared to $5 \mathrm{~d}$ but was still attenuated compared to Control. Peak responses during SOV improved further at $21 \mathrm{~d}$ and recovered by $35 \mathrm{~d}$ in all branch orders at both contraction frequencies compared to Control. (Figure 3.6 and Figure 3.7). As seen with ROV, relative responses for SOV increased with branch order and over time within each branch order. 
Recovery of active force during myofiber regeneration. Active force (g) produced by the GM when stimulated at $80-120 \mathrm{~Hz}$ (Figure 3.8) was impaired by $\sim 40 \%$ at $10 \mathrm{~d}$ vs. Control, recovered by $21 \mathrm{~d}$ and maintained at $35 \mathrm{~d}$ (Figure 3.9 - A). When normalized to muscle mass (Figure 3.9 - B), active force was significantly impaired at all time-points of regeneration vs. Control (e.g., by $47 \%$ at $10 \mathrm{~d}, 32 \%$ at $21 \mathrm{~d}$ and $27 \%$ at $35 \mathrm{~d}$ when stimulated at $100 \mathrm{~Hz}$ to evoke maximal tetanic tension). When normalized to muscle CSA, specific tension was impaired by $46 \%$ at $10 \mathrm{~d}, 31 \%$ at $21 \mathrm{~d}$ and $23 \%$ at $35 \mathrm{~d}$ vs. Control (each at $100 \mathrm{~Hz}$; Figure 3.9 - C). Muscle mass was greater than Control at all time-points during regeneration, thereby increasing muscle CSA and reducing normalized force (Table 1).

\section{DISCUSSION}

This study is the first to evaluate the time course and extent of recovery for functional vasodilation in the microvascular resistance network during skeletal muscle regeneration. Following acute injury to the $\mathrm{GM}$, elevated resting diameters with impaired vasomotor tone prevailed in all branch orders at $5 \mathrm{~d}$ yet all branches recovered to Control values by $21 \mathrm{~d}$ post injury. In response to single tetanic contraction and in response to rhythmic twitch contractions, both ROV and SOV were impaired during early regeneration (e.g. 5d), improved gradually through $10 \mathrm{~d}$ and $21 \mathrm{~d}$ and recovered by $35 \mathrm{~d}$. The active force produced by the GM was $60 \%$ of 
Control at $10 \mathrm{~d}$ post injury and recovered to Control by $21 \mathrm{~d}$. Thus, functional vasodilation required longer to recover than did force production (or vascular reactivity - see Chapter 2). I suggest that the time course for recovery of functional vasodilation begins with the formation of new muscle fibers and improves as their contractile function matures.

\section{Maximal tetanic contractions and rapid onset vasodilation}

ROV is considered an important initiating event to functional hyperemia at the onset of exercise, where muscle blood flow increases within a second following a brief contraction $(31,33,36)$. The mechanisms underlying ROV are not fully resolved. In practice, ROV occurs too quickly to be a result of an accumulation of vasoactive metabolites $(23,32,128)$. Recent findings in mice (112), hamsters (97) and humans (40) point to an integral role for the activation of $\mathrm{K}^{+}$channels in the vascular wall. Efflux of $\mathrm{K}^{+}$during repolarization of myofibers after each contraction increases interstitial $\left[\mathrm{K}^{+}\right]$, which surrounds the microvasculature. Vascular cells hyperpolarize as a result of increased $\left[\mathrm{K}^{+}\right]_{\circ}$ via activation of inward rectifying $\mathrm{K}^{+}$ channels and the $\mathrm{Na}^{+} / \mathrm{K}^{+}$ATPase in the plasma membrane $(5,32)$. Adenosine has long been known to be produced from the contraction-induced breakdown of adenine nucleotides and is formed on the extracellular side of myofibers via membrane bound ecto-5' nucleotidase $(17,18)$, thereby increasing interstitial adenosine concentration during muscle contraction. Through binding to its 
receptors on smooth muscle, adenosine plays a role in vasodilation (32) and has been implicated as a mediator of ROV if generated with sufficient rapidity (97).

Rapid onset vasodilation was impaired across branch orders during early time points of regeneration (e.g. 5d). Although improved, ROV remained attenuated at 10d (Figure 3.3) when new myofibers had formed (Figure 3.1). This may be explained by the deficit in active muscle force at $10 \mathrm{~d}$ (Figure 3.9). With maturation of regenerating myofibers at $21 \mathrm{~d}$ (Figure 3.1) [as also shown by others $(56,83)$ ], ROV improved but had not yet recovered to Control. Extending the duration of recovery to $35 \mathrm{~d}$ illustrates that ROV further improved to where this response was no longer different from Control across vessel branch orders. Although vasomotor tone recovered by $21 \mathrm{~d}$ (Figure $3.2-\mathrm{C}$ ), as did reactivity of ECs and SMCs to agonists (Chapter 2), the delay in recovery for ROV in response to GM contraction suggests that signaling between myofibers and the resistance vasculature requires additional time to recover. The signaling events underlying delayed time to peak of ROV observed especially during early regeneration time points (Figure 3.5) should be explored in future studies focused on understanding temporal aspects of ROV and its recovery during muscle regeneration.

\section{Rhythmic twitch contractions and slow onset vasodilation}

In contrast to ROV, rhythmic twitch contractions elicit vasodilation that is slower to develop, with muscle blood flow increasing gradually to a steady-state level in accord with the metabolic demand of active myofibers $(6,20,86)$. Slow onset 
vasodilation is attributable to vasodilator metabolites produced and released during muscle contractions $(32,68)$. The vasodilator nitric oxide (NO) is generated via endothelial nitric oxide synthase (eNOS) in ECs and by neuronal NOS (nNOS) found in skeletal muscle. The binding of physiological agonists such as bradykinin, acetylcholine and ATP on ECs, or an increase in luminal shear stress can stimulate the generation of NO from the endothelium, while increases in calcium in contracting myofibers can generate NO via activation of nNOS; both may contribute to SOV during rhythmic twitch contractions $(32,68,101)$. Vasodilator prostanoids such as prostaglandin and prostacyclin are also produced in myofibers and ECs, which can then act on SMCs to cause relaxation and vasodilation to increase muscle blood flow during exercise $(32,101)$.

As observed for ROV in response to tetanic contraction, SOV in response to rhythmic twitch contractions at $2 \mathrm{~Hz}$ and $4 \mathrm{~Hz}$ was also impaired during early regeneration at $5 d$ post injury across branch orders (Figure 3.6 and Figure 3.7). SOV improved progressively during regeneration as new myofibers formed and matured $(56,67,83)$ (Figure 3.1) such that by $35 \mathrm{~d}$ post injury, SOV was not different from Control. Collectively, these data provide the first timeline for recovery of functional vasodilation while illustrating that SOV and ROV recover with a similar time course during skeletal muscle regeneration after acute injury. For both types of contraction, the absolute changes in diameter were similar across branch orders. However, when expressed relative to maximal diameter, vasodilation increased with branch order as vessels decreased in size. For all branch orders, 
functional vasodilation improved with time during recovery, indicating that irrespective of the underlying signaling events for ROV vs SOV, the ability of the resistance vasculature to respond to myofiber contraction follows a similar time course of recovery.

\section{Active force during skeletal muscle regeneration}

Muscle function is recovered when new muscle fibers are formed, matured and reinnervated $(30,83,96)$. To underscore my studies of how functional vasodilation recovers during regeneration, I evaluated the time course and extent for restoration of active force production by $\mathrm{GM}$ myofibers after $\mathrm{BaCl}_{2}$ injury. Since muscle fibers were just beginning to regenerate at $5 \mathrm{~d}$ with many fibers still damaged, active force was expected to be negligible and was therefore not evaluated until fiber integrity was restored (at 10d). When new GM muscle fibers were clearly present at 10d, maximum tetanic force $(\mathrm{g})$ was $\sim 60 \%$ of Control and recovered to Control by $21 \mathrm{~d}$, when myofibers had enlarged and appeared similar to Control in size. Active force was maintained at $35 d$, confirming recovery of the GM in this capacity (Figure $\mathbf{3 . 9}$ - A). However, when normalized to muscle mass (g force/g muscle) (Figure 3.9 B) or to muscle cross-sectional area $\left(\mathrm{CSA}, \mathrm{mN} / \mathrm{mm}^{2}\right)$ (Figure $3.9-\mathrm{C}$ ), maximal force plateaued at $\sim 75 \%$ of Control at 21 and $35 \mathrm{~d}$. The increase in muscle mass and CSA (Table 1) in the regenerating GM contributed to the decrement observed in normalized active force at respective time points of recovery. Since muscle mass and total CSA of a given muscle includes intracellular and extracellular tissue components (along with changes in composition), changes in fluid content, 
connective tissue and intracellular total protein content contribute to changes in muscle mass and cross section area. A previous study demonstrated that decreased specific tension in soleus muscle grafts (108) was a result of increased fluid content and noncontractile tissue components. Therefore, factors such as increased fluid content and noncontractile tissue components could potentially be contributing to increased muscle mass and decreased normalized active force observed in my study at 21 and $35 \mathrm{~d}$ post injury compared to Control. Further studies are needed to resolve the nature of tissue composition in the regenerating GM, particularly when active force has stabilized. The ability of regenerated muscle to sustain activity (i.e., fatigability) should also be defined as a reference for therapeutic intervention aimed at restoring muscle function in vivo.

\section{SUMMARY AND CONCLUSION}

Using a novel muscle injury model in evaluating functional vasodilation at different contraction durations and contraction frequencies, this study for the first time provides novel insight into when and to what extent functional vasodilation and muscle active force recover in the GM during regeneration following acute injury. The need for additional recovery time for functional vasodilation (35 days) compared to recovery of vasomotor tone ( 21 days) suggests that signaling between muscle fibers and resistance vessels during vasodilation in response to muscle contraction takes longer to recover than does vasomotor tone and vascular reactivity to pharmacological agonists (Chapter 2). Despite markedly different signaling pathways, ROV and SOV recovered in parallel, and although total active 
force recovered by $21 d$, specific tension was still impaired at $35 d$, requiring future studies to determine the basis of such differences. The findings in this study point to how ROV and SOV recover during regeneration, when new myofibers are formed and their function is regained and provides a reference for when physical activity can be resumed post muscle injury. Future studies are needed to understand mechanisms underlying impaired functional vasodilation and active force with injury to the GM, which will advance our understanding of recovery of muscle blood flow in contracting muscle and designing of future therapeutic interventions in facilitating recovery. 


\section{FIGURES}

\section{Control}

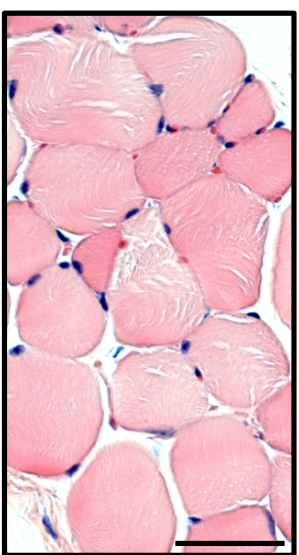

$5 d$

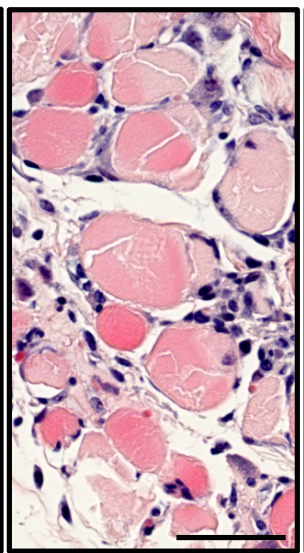

10 21

35

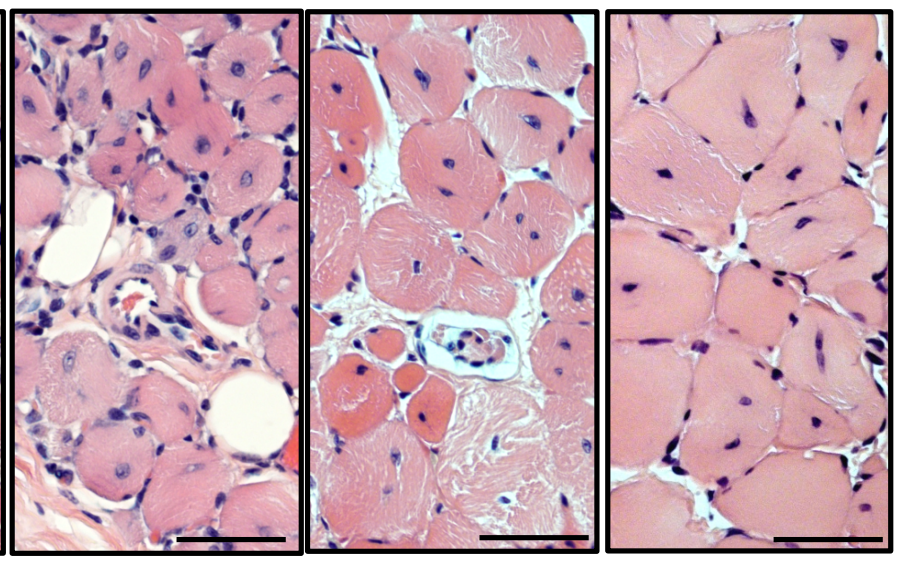

Figure 3. 1. Hematoxylin and eosin staining of uninjured GM muscle fibers (Control) and regenerating $\mathrm{GM}$ muscle fibers at $5,10,21$ and $35 \mathrm{~d}$ after $\mathrm{BaCl}_{2}$ injury.

Note damaged muscle fibers at $5 \mathrm{~d}$ and regenerating muscle fibers (with centrally located nuclei) at $10 \mathrm{~d}$. Regenerated muscle fibers at $21 \mathrm{~d}$ and $35 \mathrm{~d}$ retain central nuclei. Scale bars $=50 \mu \mathrm{m}$. 
A

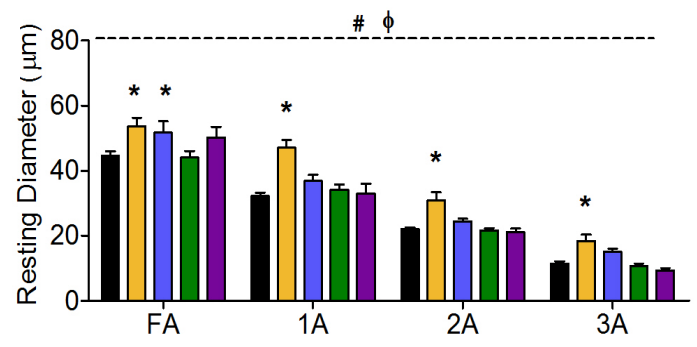

B

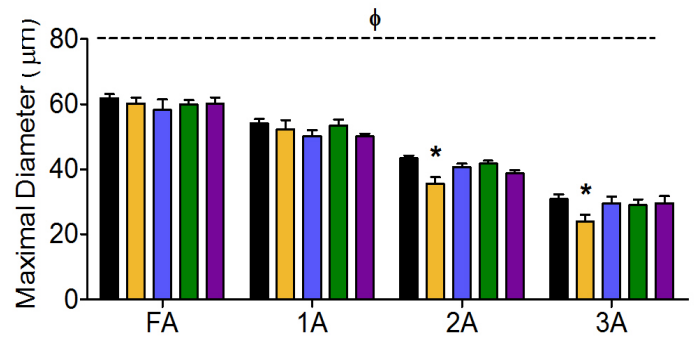

$C$

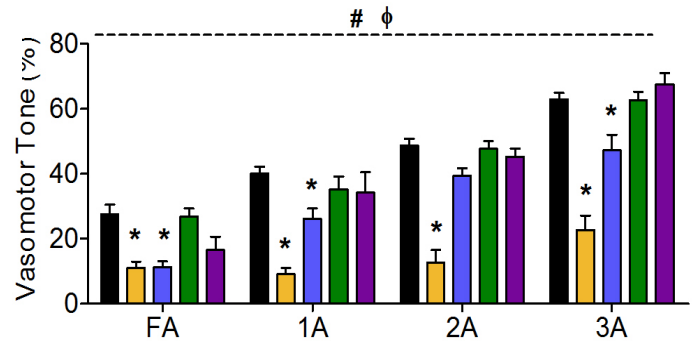

Figure 3. 2. Resting and maximal diameters and vasomotor tone for FA, $1 A, 2 A$ and $3 A$ during regeneration of the $\mathbf{G M}$.

(A) Following GM injury with $\mathrm{BaCl}_{2}$, resting diameters increased at $5 \mathrm{~d}$ and recovered by $21 \mathrm{~d}$ in all vessel branch orders. (B) Maximal diameters (sodium nitroprusside, $10^{-4} \mathrm{M}$ ) were not different across time except in $2 \mathrm{~A}$ and $3 \mathrm{~A}$ at $5 \mathrm{~d}$ post injury. (C) Spontaneous vasomotor tone decreased at $5 \mathrm{~d}$ and recovered by $21 \mathrm{~d}$ in all vessel branch orders. Summary data are means \pm S.E.; $n=5$ per group. ${ }^{\#} P<0.05$, effect of time. ${ }^{\Phi} \mathrm{P}<0.05$, effect of branch order. ${ }^{*} \mathrm{P}<0.05$ vs Control. 

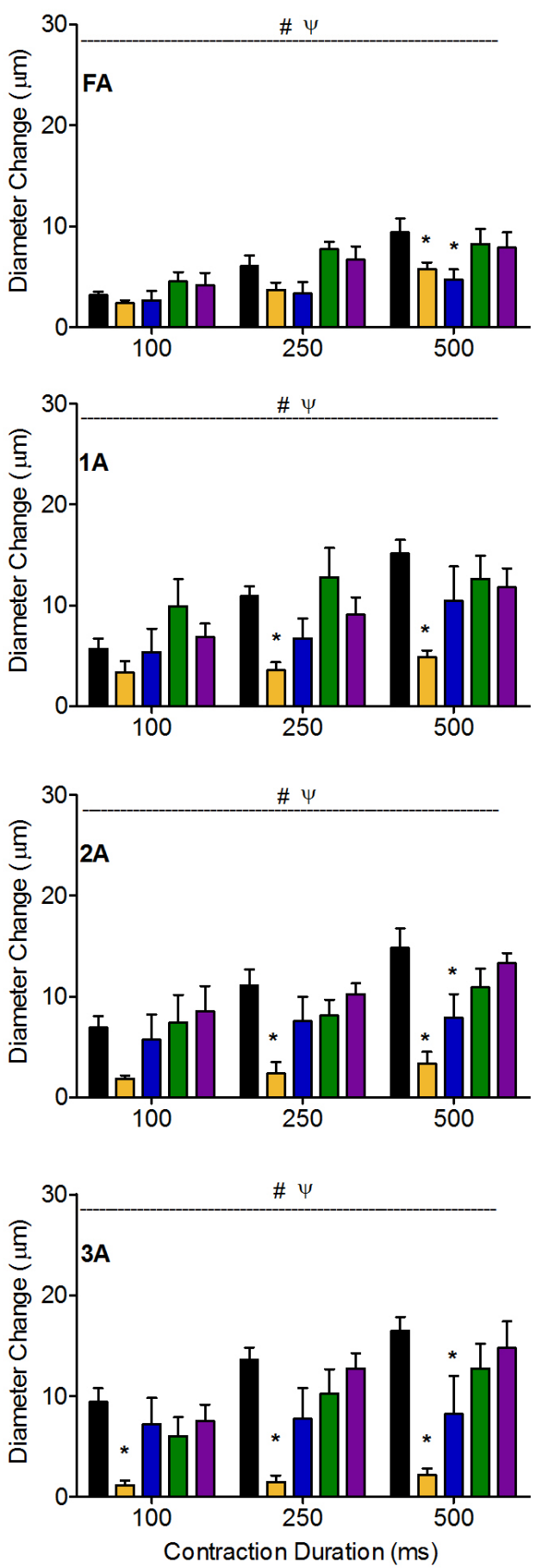

Figure 3. 3. Rapid onset vasodilation (ROV) during muscle regeneration: Diameter change

In response to a tetanic contraction (100 $\mathrm{Hz}$ ) for 100,250 or $500 \mathrm{~ms}$ duration, ROV was impaired at $5 \mathrm{~d}$ in all branch orders, improved progressively at $10 \mathrm{~d}$ and $21 \mathrm{~d}$, and was similar to control at $35 \mathrm{~d}$. Summary data are means \pm S.E.; $n=5$ per group. ${ }^{\#} P<0.05$, effect of time. ${ }^{*} P<0.05$, effect of contraction duration. ${ }^{*} \mathrm{P}<0.05$ vs Control. 


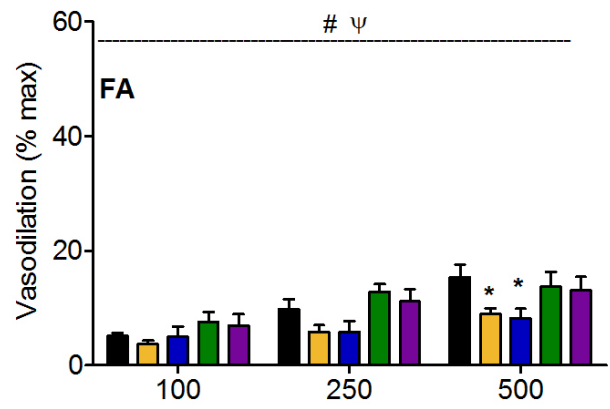

Figure 3. 4. Rapid onset vasodilation (ROV) during muscle regeneration: Normalized data

In response to a tetanic contraction (100 $\mathrm{Hz}$ ) for 100,250 or 500 ms duration, ROV was impaired at $5 d$ in all branch orders, improved progressively at $10 \mathrm{~d}$ and $21 \mathrm{~d}$, and was similar to control at $35 \mathrm{~d}$. Summary data are means \pm S.E.; $n=5$ per group. ${ }^{\#} \mathrm{P}<0.05$, effect of time. ${ }^{\psi} \mathrm{P}<0.05$, effect of

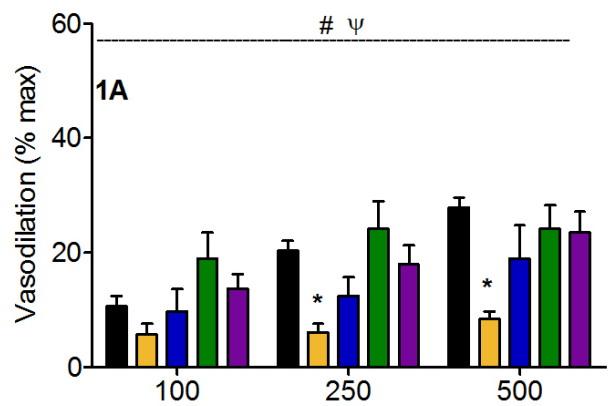
contraction duration. ${ }^{*} \mathrm{P}<0.05$ vs Control.
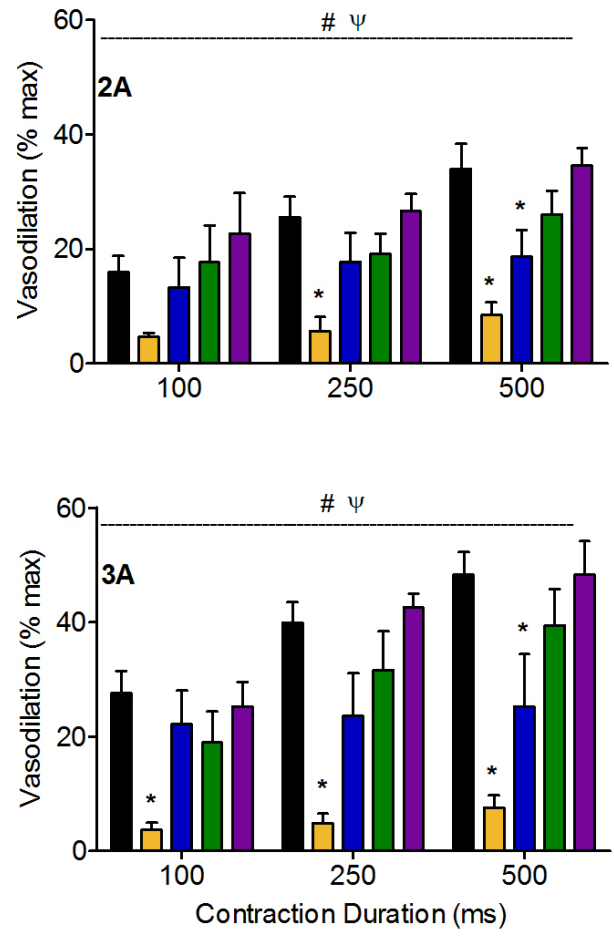
$\rightarrow$ Control $\prec 5 \mathrm{~d} \rightarrow 10 \mathrm{~d} \rightarrow 21 \mathrm{~d} \rightarrow 35 \mathrm{~d}$

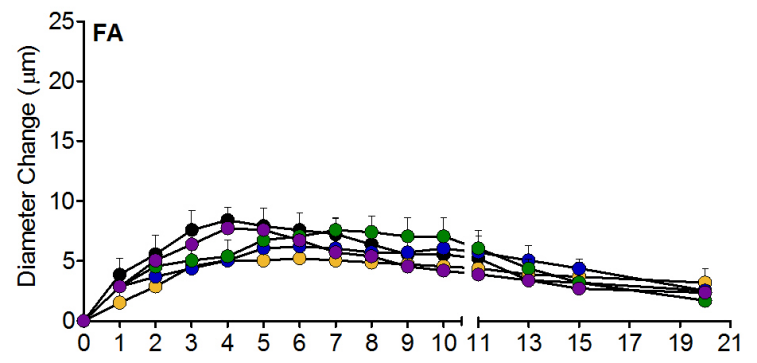

Figure 3. 5. Time course of Rapid onset vasodilation (ROV) at $500 \mathrm{~ms}$ contraction duration.

In response to a tetanic contraction (100 $\mathrm{Hz}, 500 \mathrm{~ms}$ ), the time course and amplitude of ROV were attenuated at $5 d$ in all branch orders, then became progressively greater and faster at $10 \mathrm{~d}$, $21 \mathrm{~d}$, and $35 \mathrm{~d}$. Summary data are means \pm

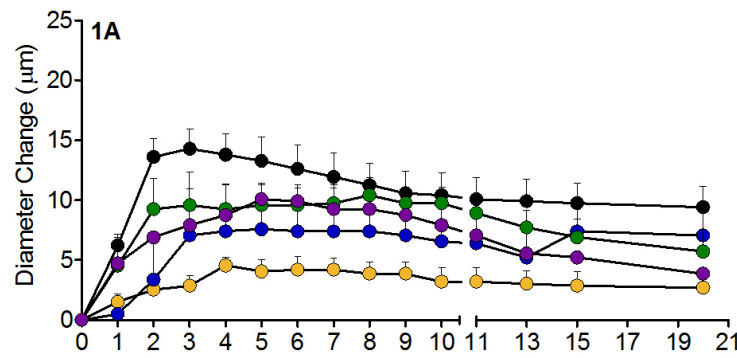
S.E.; $n=5$ per group.
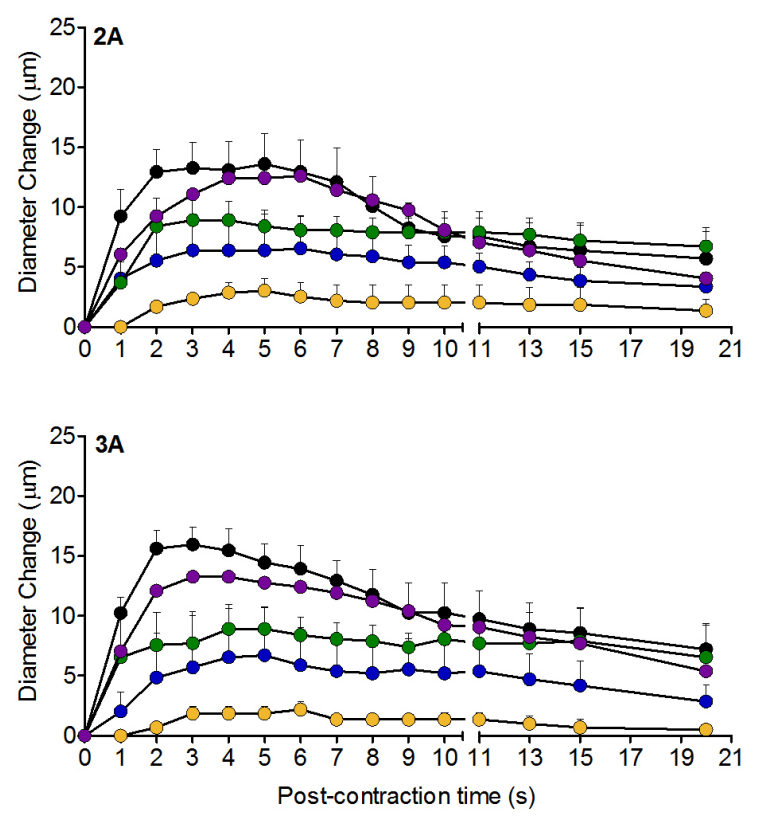
Control $5 d$

\# $\psi$
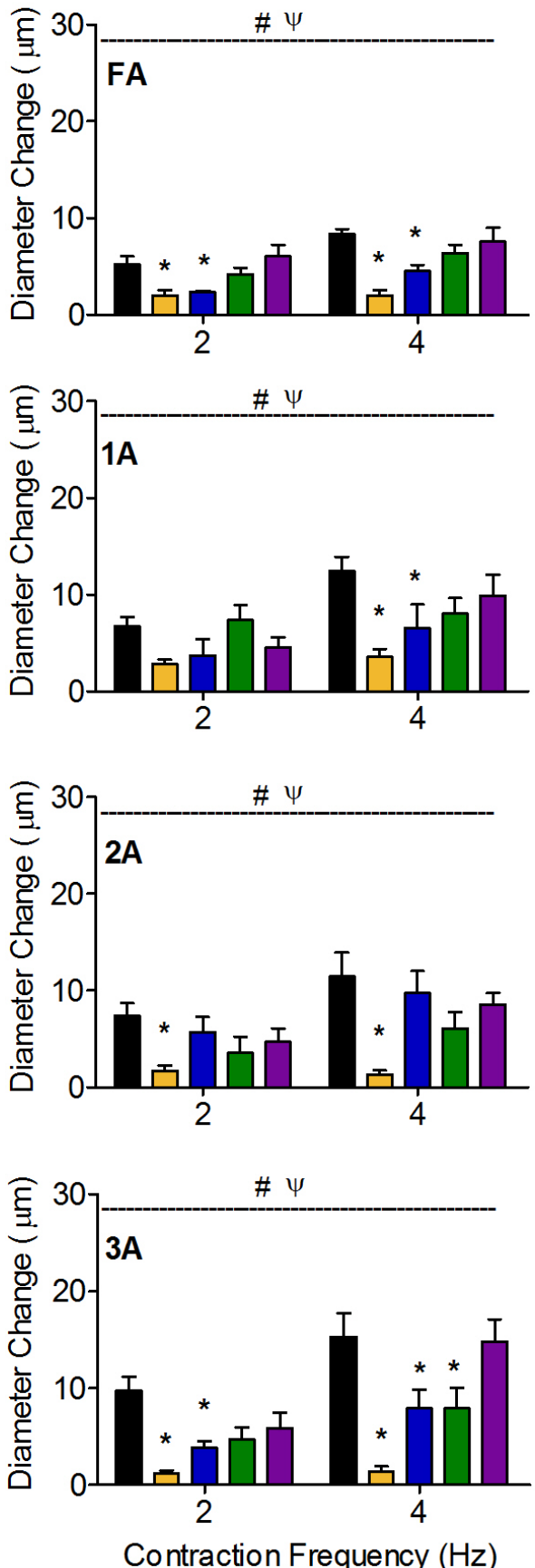

Figure 3. 6. Slow onset vasodilation (SOV) during muscle regeneration: Diameter change

In response to rhythmic twitch contractions at $2 \mathrm{~Hz}$ and $4 \mathrm{~Hz}$ for $30 \mathrm{~s}$, SOV was impaired at $5 \mathrm{~d}$ in all branch orders, improved progressively at $10 \mathrm{~d}$ and $21 \mathrm{~d}$, and was similar to control at $35 \mathrm{~d}$. Summary data are means \pm S.E.; $n=5$ per group. $\# P<0.05$, effect of time. $\psi P<<0.05$, effect of contraction frequency. ${ }^{*} \mathrm{P}<0.05$ vs Control. 
Control $\square$ 5d $\square$ 10d $\square$ 21d $\square$ 35d

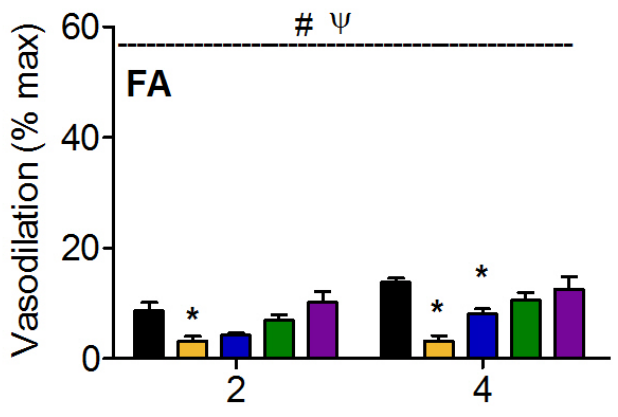

Figure 3. 7. Slow onset vasodilation (SOV) during muscle regeneration: Normalized data

In response to rhythmic twitch contractions at $2 \mathrm{~Hz}$ and $4 \mathrm{~Hz}$ for $30 \mathrm{~s}$, SOV was impaired at $5 \mathrm{~d}$ in all branch orders, improved progressively at $10 \mathrm{~d}$ and $21 \mathrm{~d}$, and was similar to control at $35 \mathrm{~d}$. Summary data are means \pm S.E.; $n=5$ per group. $\# P<0.05$, effect of time. $\psi P<<0.05$, effect of contraction frequency. ${ }^{*} \mathrm{P}<0.05$ vs Control.
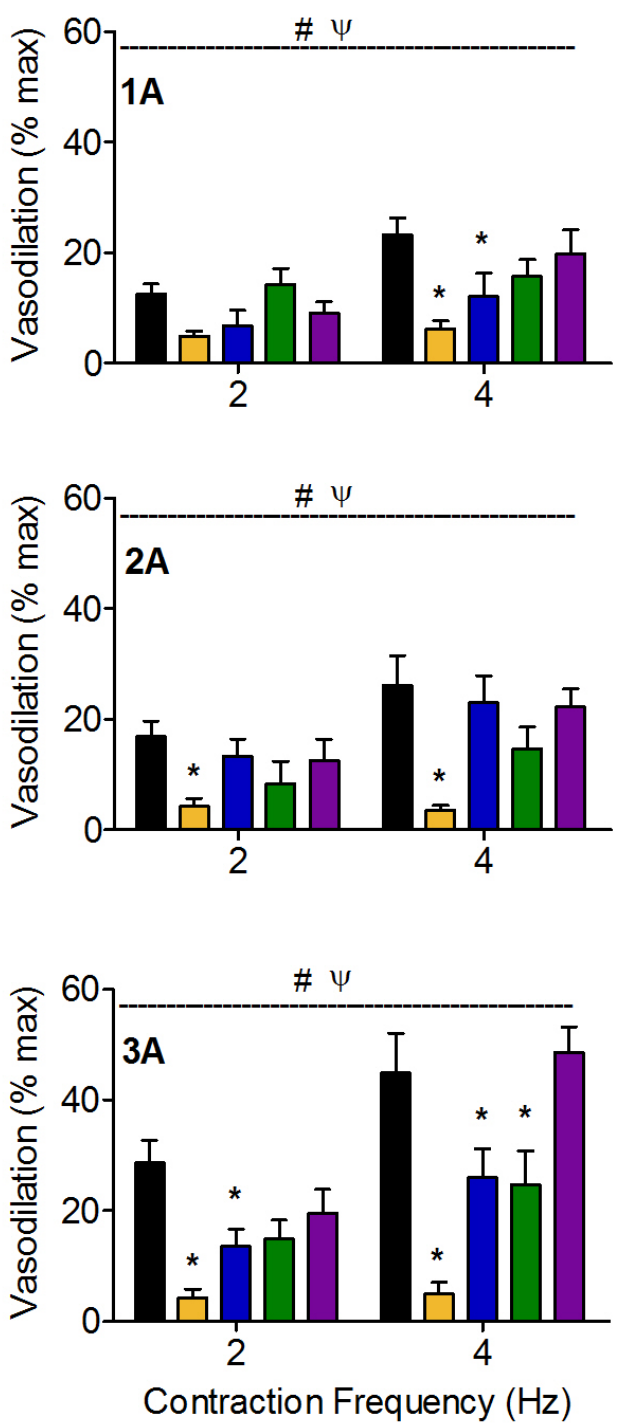


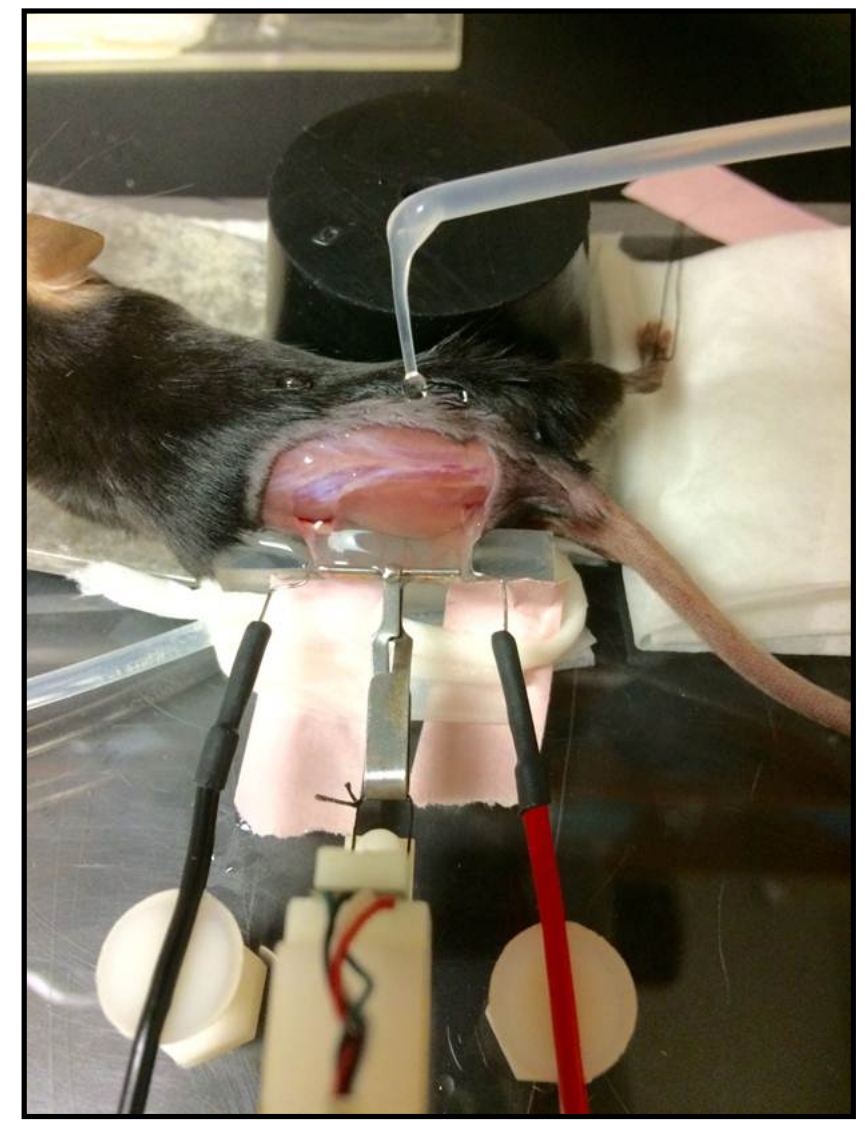

Figure 3. 8. Preparation of the GM for active force measurements.

Platinum wire electrodes are positioned on either side of the muscle for electrical field stimulation with the GM secured in an atraumatic clamp connected to a force transducer while continuously irrigated with PSS. 


\begin{tabular}{|c|c|c|c|c|}
\hline & Control & 10d & 21d & 35d \\
\hline Muscle Mass & $54.2 \pm 2.3$ & ${ }^{*} 66.6 \pm 7.6$ & ${ }^{*} 76.7 \pm 3.2$ & ${ }^{*} 70.8 \pm 2.8$ \\
\hline CSA $\left(\mathrm{mm}^{2}\right)$ & $5.1 \pm 0.2$ & ${ }^{*} 6.2 \pm 0.6$ & ${ }^{*} 7.2 \pm 0.4$ & ${ }^{*} 6.4 \pm 0.4$ \\
\hline
\end{tabular}

Table 1. Muscle mass and Cross-Sectional Area (CSA) of the GM during regeneration at 10d, 21d and 35d compared to Control.

Summary data are means \pm S.E.; $n=5$ per group. Mass and CSA were greater $\left({ }^{*} P<0.05\right)$ at $10 \mathrm{~d}$, $21 \mathrm{~d}$ and $35 \mathrm{~d}$ vs Control. ( $\mathrm{n}=5 /$ group). CSA $=$ Muscle mass / (Muscle Length $\mathrm{X}$ Density)]. 
A
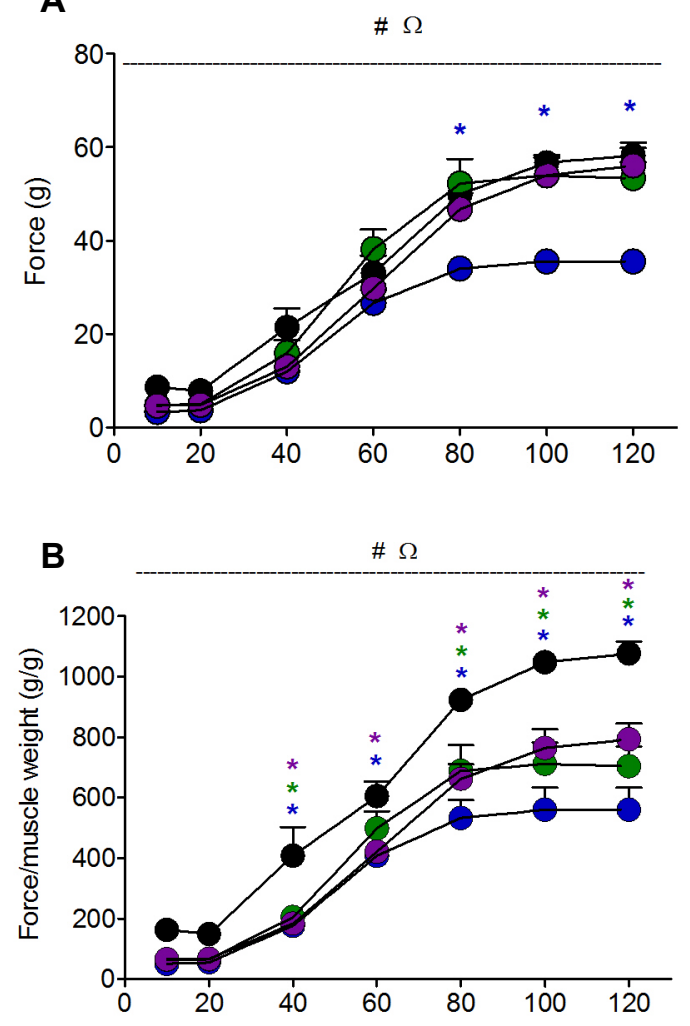

C

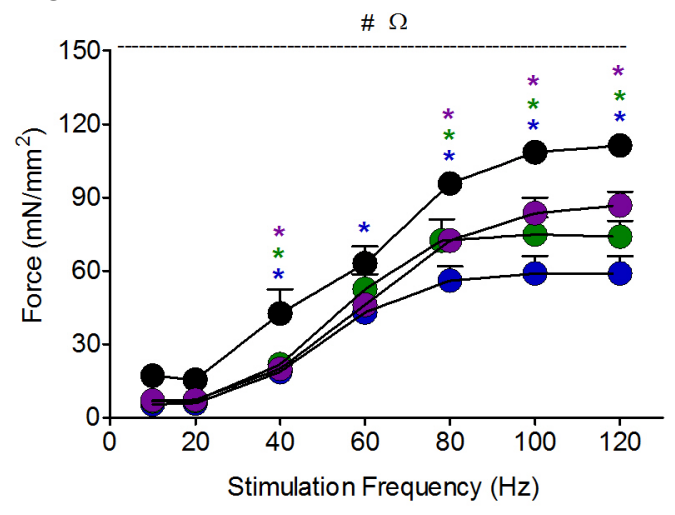

Figure $3 . \quad 9$ Frequency-force relationships during regeneration of the GM.

(A) Maximal active force (g) during electrical stimulation was $\sim 60 \%$ of Control at $10 \mathrm{~d}$, recovered by $21 \mathrm{~d}$ and was maintained through $35 \mathrm{~d}$. Normalized to $(B)$ muscle mass $(\mathrm{g} / \mathrm{g})$ or to $(\mathrm{C})$ cross-sectional area (CSA, $\mathrm{mN} / \mathrm{mm}^{2}$ ), maximal force plateaued at $\sim 75 \%$ of Control during regeneration. Summary data are means \pm S.E.; $n=5$ per group. ${ }^{\#} \mathrm{P}<0.05$, effect of time. $\Omega \mathrm{P}<0.05$, effect of stimulation frequency. ${ }^{*} \mathrm{P}<0.05$ vs Control. 


\section{CHAPTER 4}

\section{DISCUSSION}

\section{SUMMARY}

My dissertation research has investigated when blood flow is restored and becomes functionally regulated in regenerating skeletal muscle following injury. To investigate the functional recovery of the microcirculation in vivo using intravital microscopy, I developed a new model of acute injury using the mouse GM. I used local injection of $\mathrm{BaCl}_{2}$ to kill myofibers and to then evaluate recovery of vasomotor responses of the microvascular resistance network in response to to physiological agonists and to functional vasodilation in response to muscle contraction. To understand whether recovery of microvascular function parallels the recovery of skeletal muscle function, I evaluated active force produced by the GM at the key time-points of study defined by my experiments. These physiological measurements enabled me to determine when and to what extent the coupling between contractile function and functional vasodilation recover during regeneration following acute injury to skeletal muscle in the mouse.

Chapter 2 describes the application of my GM injury model to study the recovery of microvascular perfusion and vasomotor responses to pharmacological agonists in regulating blood flow to skeletal muscle. By injecting $75 \mu$ of a $1.2 \% \mathrm{BaCl}_{2}$ solution under the GM, I was able to kill myofibers and damage their microvascular 
networks including the feed artery (defined anatomically as the inferior gluteal artery) and arterioles that control blood flow and the capillaries surrounding each myofiber. Chapter 2 answers the key question: When is tissue perfusion restored during regeneration following acute injury? I administered a fluorescent probe, a $70 \mathrm{kDa}$ FITC-dextran, via a retro-orbital injection to label plasma systemically and by observing the regenerating microcirculation. I found that tissue perfusion was restored by $5 \mathrm{~d}$ post injury by confirming fluorescent plasma within the arteriolar network and capillaries. Histologically, tissue cross-sections labeled with embryonic myosin heavy chain revealed initiation of muscle regeneration at $5 \mathrm{~d}$, completion of new fiber formation at $10 \mathrm{~d}$ and mature myofibers at $21 \mathrm{~d}$ post injury that were similar in size to Control myofibers. Therefore, these time-points were selected in studying the recovery of the microcirculation to determine when and to what extent vasomotor control recovered during respective stages of myofiber regeneration.

I found that spontaneous vasomotor tone was impaired at $5 \mathrm{~d}$ and $10 \mathrm{~d}$ post injury, attributable to a significant increase in resting diameter of $F A, 1 A, 2 A$ and $3 A$ branch orders of the resistance network; maximal diameters were generally not different from Control. Spontaneous vasomotor tone recovered by $21 \mathrm{~d}$, suggesting that mechanisms regulating vasomotor tone are recovered when myofibers have matured. Endothelium-dependent vasodilation, evaluated by recording diameter responses to increasing concentrations of ACh applied topically, was also impaired at $5 \mathrm{~d}$ in all branch orders. However, by $21 \mathrm{~d}$, endothelium-dependent vasodilation 
recovered and was no longer different from Control (Figure 4.1). Interestingly, differences in the recovery of the branch orders were observed at $10 \mathrm{~d}$, where smaller distal $2 \mathrm{~A}$ and $3 \mathrm{~A}$ branches recovered sooner than larger proximal $\mathrm{FA}$ and $1 \mathrm{~A}$, suggesting a distal-to-proximal gradient in the recovery of endotheliumdependent vasodilator signaling. This leads me to suggest that vessel structures (endothelial and smooth muscle cell layers) of smaller branches recover sooner when compared to larger arterioles and FA, therefore their function in regulating regional flow distribution recovers sooner than the ability to regulate total blood flow entering the muscle. Sympathetic vasoconstriction to cumulative increases in the concentration of the $\alpha_{1}$ adrenoreceptor stimulation with PE was also impaired at $5 \mathrm{~d}$ in all branch orders. At $10 \mathrm{~d}$, all branch orders exhibited constriction with PE and by $21 \mathrm{~d}$ sympathetic vasoconstriction was no longer different from Control. Collectively, my data suggest that spontaneous vasomotor tone, endotheliumdependent vasodilation and sympathetic vasoconstriction recover gradually and progressively in concert at each stage of muscle regeneration.

Chapter 3 describes the recovery of functional vasodilation during skeletal muscle regeneration following $\mathrm{BaCl}_{2}$ injury in $\mathrm{FA}, 1 \mathrm{~A}, 2 \mathrm{~A}$ and $3 \mathrm{~A}$ branch orders of the $\mathrm{GM}$ resistance network. In these experiments, I evaluated the hyperemic response to varying intensities and durations of myofiber contraction (i.e., metabolic demand) at $5 d, 10 d, 21 d$ and $35 d$ compared to uninjured Control. To simulate a brief, intense increase in metabolic demand, ROV was evaluated by contracting the GM with single tetanic contractions at $100 \mathrm{~Hz}$ with at durations of 100,250 and $500 \mathrm{~ms}$. I 
found that ROV was impaired at $5 \mathrm{~d}$ post injury in all branch orders. Although showing improvement, ROV was still impaired at 10d (vs Control) and improved further by $21 \mathrm{~d}$ post injury in all branch orders across contraction durations. However, ROV was not restored to Control by $21 \mathrm{~d}$, so I extended the period of recovery and evaluated ROV at 35d post injury. My data from this later time point indicate that ROV had recovered in all branch orders. To simulate a moderate, sustained increase in metabolic demand, slow onset vasodilation was evaluated by contracting the GM with rhythmic twitch contractions at 2 and $4 \mathrm{~Hz}$ for $30 \mathrm{~s}$ and peak dilation was measured in all branch orders at $5 d, 10 d, 21 d$ and $35 d$ post injury. I found that SOV was impaired at $5 \mathrm{~d}$ in all branch orders and improved progressively at $10 \mathrm{~d}$ and $21 \mathrm{~d}$, with recovery to Control values at $35 \mathrm{~d}$ post injury. Thus, SOV and ROV appear to recover in parallel despite differences in their signaling pathways.

My findings show that, while spontaneous vasomotor tone and the reactivity of ECs and SMCs to physiological agonists had recovered by $21 \mathrm{~d}$, functional vasodilation in response to myofiber contraction required more time to restore the signaling events involved in the hyperemic responses to different exercise intensities (Figure 4.1). Histological analysis (hematoxylin \& eosin staining) of GM crosssections following $\mathrm{BaCl}_{2}$ injury showed extensive damage to myofibers at $5 \mathrm{~d}$ with a few having central nuclei; new myofiber formation was pronounced throughout the muscle at $10 \mathrm{~d}$ as confirmed by their having centralized nuclei. These newlyformed myofibers increased in size at $21 \mathrm{~d}$ and $35 \mathrm{~d}$, showing maturation of the 
newly regenerated muscle when embryonic myosin was no longer being expressed (Figure 2.3).

Recovery of muscle force production was evaluated by contracting the GM at increasing frequencies from $10 \mathrm{~Hz}$ to $120 \mathrm{~Hz}$ in $20 \mathrm{~Hz}$ increments and measuring active force generated at the respective contraction frequencies. Since muscle fibers were just beginning to regenerate at $5 \mathrm{~d}$, force measurements were not evaluated until $10 \mathrm{~d}$, with additional studies at $21 \mathrm{~d}$ and $35 \mathrm{~d}$. Compared to Control, active force (as measured in grams) was impaired at $10 \mathrm{~d}$, recovered by $21 \mathrm{~d}$ and was maintained at $35 \mathrm{~d}$ (Figure 4.1). When active force was normalized to muscle weight or cross-sectional area, active force recovered to $\sim 75 \%$ of control at $21 \mathrm{~d}$ and remained at this level through $35 \mathrm{~d}$. In light of the greater weight of the regenerated GM compared to Control, my evaluation of active force production implies that increased fluid content, non-contractile tissue components and/or decreased intracellular protein content may contributing to the increase in muscle mass and decrease in normalized active force observed at 21 and $35 \mathrm{~d}$ post injury compared to Control. My findings in chapter 3 suggest that although vasomotor tone recovers by $21 \mathrm{~d}$, additional time is needed for recovery of ROV and SOV as well as recovery of specific tension of the GM. 


\section{IMPLICATIONS OF CURRENT FINDINGS}

Understanding the time course and extent for the recovery of blood flow regulation by the endothelium and smooth muscle of the resistance microvasculature provides novel insight needed for understanding the recovery of signaling mechanisms involved in endothelium-dependent vasodilation and sympathetic vasoconstriction at each phase of muscle regeneration studied following injury. Impairment in vasomotor tone observed with injury and during early regeneration at $5 \mathrm{~d}$ can now be targeted in understanding what factors contribute to this impairment and develop effective strategies in facilitating recovery of vasomotor tone and thereby accelerating recovery of blood flow regulation in regenerating skeletal muscle. Current research has identified delivery of growth factors $(13,22,60,74,131)$ as well as adult and pluripotent stem cells $(50,72,76,77,130)$ as treatment options in facilitating skeletal muscle recovery from injury. Therefore, understanding when and to what extent the microcirculation recovers during the different phases of regeneration provides important new information applicable to selecting appropriate treatment options in facilitating recovery.

Understanding when and to what extent the coupling between contractile function and functional vasodilation recover during regeneration following skeletal muscle injury will also provide insight into signaling mechanisms that are impaired with injury and the time required for their recovery. The data from my research will therefore aid in understanding when physical activity, especially in athletes where 
tissue perfusion is crucial in meeting the metabolic demands of championship performance, can be resumed after injury. Studies have investigated the role of exercise/contractile activity as therapy after skeletal muscle injury and shown how exercise increases skeletal muscle mass and CSA of regenerating muscle $(69,93$, 94). Exercise is known to initiate transcription of antioxidant genes and specific signaling pathways that results in increased production of antioxidant enzymes that could facilitate muscle remodeling (73). Therefore, the data from my research provide a time course with respect to when muscle function and functional vasodilation mechanisms are recovered and when physical activity can be resumed to further facilitate recovery of skeletal muscle during regeneration following acute injury. Further, these data provide a reference for investigating how signaling events involved in recovery of the microcirculation during regeneration are affected during cardiovascular disease (e.g., diabetes and advanced age), and how recovery of microvascular function may be prolonged in such disease states.

\section{FUTURE DIRECTIONS}

Future studies should be aimed at investigating the extent of structural damage to the GM microvascular network with $\mathrm{BaCl}_{2}$ injury, especially in the $\mathrm{FA}, 1 \mathrm{~A}, 2 \mathrm{~A}$ and $3 \mathrm{~A}$ branch orders of the resistance network. Of particular interest is the structure and integrity of endothelial and smooth muscle layers of these branch orders during the different phases of regeneration. Because structure defines function, investigating structural recovery of the microvasculature will provide critical insight 
into trends of functional recovery apparent from my research. For example, it would be important to identify whether elevated vasomotor tone during early regeneration at $5 d$ is due to smooth muscle structural damage and loss of contractility versus increased production of NO or other vasodilator agents.

With injury and inflammation, iNOS is expressed by macrophages in damaged and regenerating skeletal muscle to regulate and facilitate myogenic precursor cells in the healing process (95). The resulting generation of NO via activation of iNOS appears to be required for the formation of new myofibers $(3,95,117)$ and could potentially be contributing to vasodilation and impaired vasomotor tone. Therefore, future studies could be aimed at inhibiting macrophages during skeletal muscle regeneration following injury. These experiments would determine whether NO generation via activation of iNOS contributes to impaired vasomotor tone. Because iNOS expressed in macrophages also facilitates myogenic precursor cell function during regeneration, inhibiting macrophages could impair skeletal muscle regeneration. With my data for reference, appropriate experiments can now be designed to address these key relationships.

The arteriolar branches of the microcirculation are innervated by the sympathetic nervous system and sympathetic nerve activity can elevate vasomotor tone (61, 68). Therefore, future studies could be designed in labeling perivascular sympathetic nerves at the different phases of regeneration, especially during early phases of regeneration, in determining whether loss of sympathetic tone 
contributes to impaired tone and resulting blood flow regulation. These experiments will provide valuable insight as to why vasomotor functions are impaired immediately following injury and during early regeneration when myofibers are beginning to regenerate. One hypothesis that could be readily tested using immunostaining and perivascular nerve stimulation is that restoration of vasomotor tone accompanies regeneration of perivascular sympathetic nerves.

With respect to recovery of vasodilation during skeletal muscle regeneration and understanding signaling mechanisms involved in ascending vasodilation of $1 \mathrm{~A}$ and FA branches that results in increased blood flow into skeletal muscle, future studies could be aimed at understanding when and to what extent these signaling mechanisms recover during regeneration. Feed arteries of the hamster retractor muscle have been used previously in demonstrating two distinct and complementary signaling pathways involved in SMC relaxation to focal delivery of ACh (44). The first was recognized as electromechanical conduction of vasodilation with spread of hyperpolarization along the endothelium and into SMCs via activation of $\mathrm{IK}_{\mathrm{Ca}}$ and $\mathrm{SK}_{\mathrm{Ca}}$ channels in ECs. The second was identified as pharmacomechanical conduction mediated by $\mathrm{Ca}^{2+}$ waves and the release of autocoids (e.g. NO and $\mathrm{PGI}_{2}$ ) along the endothelium (44). Recent studies (112) have also shown that vasodilation ascends from downstream arterioles into upstream FAs in response to GM contraction by both rapid and slow onset signaling, thereby demonstrating physiological relevance of both signaling pathways during exercise. Therefore, using isolated arteries and/or arterioles, or 
the intact microcirculation in response to GM contraction, it would be interesting to explore these mechanisms in understanding when ascending vasodilation recovers following skeletal muscle injury.

My dissertation research studies have identified fundamental relationships between myofibers and microvessels during skeletal muscle regeneration and have provided a new foundation in raising future mechanistic questions in determining recovery of blood flow regulation in regenerating skeletal muscle. 


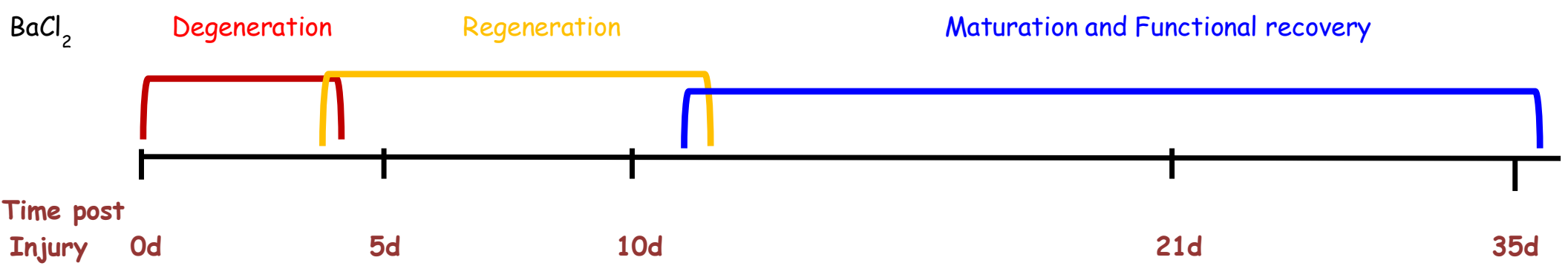

$\begin{array}{llll}\text { Vasomotor tone } & \text { Impaired } & \text { Improved } & \text { Recovered } \\ \text { EDD } & \text { Impaired } & \text { Improved } & \text { Recovered } \\ \text { Vasoconstriction }\left(\alpha_{1}\right) & \text { Impaired } & \text { Improved } & \text { Recovered } \\ \text { Functional vasodilation } & \text { Impaired } & \text { Improved } & \text { Improved } \\ \text { Active force }(g) & \mathrm{NA} & \text { Impaired } & \text { Recovered Recovered }\end{array}$

Figure 4. 1. Recovery of vasomotor responses and muscle function during skeletal muscle regeneration following injury.

Following $\mathrm{BaCl}_{2}$ injury of the $\mathrm{GM}$, initial regeneration of myofibers began at $\sim 5 \mathrm{~d}$ post injury. Vasomotor tone, endothelium-dependent dilation (EDD), sympathetic vasoconstriction and functional vasodilation were impaired during initial regeneration at $5 \mathrm{~d}$. Vasomotor responses improved at $10 \mathrm{~d}$ when new myofibers were formed and initial regeneration nearing completion. Vasomotor tone, EDD and sympathetic vasoconstriction recovered by $21 \mathrm{~d}$ during maturation of newly formed muscle fibers. Although improved at $21 \mathrm{~d}$, functional vasodilation recovered at $35 \mathrm{~d}$. Muscle active force $(\mathrm{g})$ was impaired at $10 \mathrm{~d}$, however it recovered by $21 \mathrm{~d}$ and recovery was maintained at $35 \mathrm{~d}$ post injury. 


\section{REFERENCES}

1. Aaker A, and Laughlin MH. Diaphragm arterioles are less responsive to $\alpha_{1}$-adrenergic constriction than gastrocnemius arterioles. J Appl Physiol 92: 1808-1816, 2002.

2. Adair TH MJ. Angiogenesis. Morgan \& Claypool Life Sciences 2010.

3. Anderson JE. A role for nitric oxide in muscle repair: nitric oxide-mediated activation of muscle satellite cells. Mol Biol Cell 11: 1859-1874, 2000.

4. Archer SL, Huang JM, Hampl V, Nelson DP, Shultz PJ, and Weir EK. Nitric oxide and cGMP cause vasorelaxation by activation of a charybdotoxin-sensitive $\mathrm{K}$ channel by cGMP-dependent protein kinase. Proc Natl Acad Sci U S A 91: 7583-7587, 1994.

5. Armstrong $\mathbf{M L}$, Dua $\mathbf{A K}$, and Murrant $\mathrm{CL}$. Potassium initiates vasodilatation induced by a single skeletal muscle contraction in hamster cremaster muscle. J Physiol 581: 841-852, 2007.

6. Armstrong RB, and Laughlin $\mathbf{M H}$. Rat muscle blood flows during highspeed locomotion. J Appl Physiol 59: 1322-1328, 1985.

7. Armstrong RB, Warren GL, and Warren JA. Mechanisms of exerciseinduced muscle fibre injury. Sports Med 12: 184-207, 1991.

8. Arpino JM, Nong Z, Li F, Yin H, Ghonaim N, Milkovich S, Balint B, O'Neil C, Fraser GM, Goldman D, Ellis CG, and Pickering JG. FourDimensional Microvascular Analysis Reveals That Regenerative Angiogenesis in Ischemic Muscle Produces a Flawed Microcirculation. Circ Res 120: 1453-1465, 2017.

9. Arsic N, Zacchigna S, Zentilin L, Ramirez-Correa G, Pattarini L, Salvi A, Sinagra G, and Giacca M. Vascular endothelial growth factor stimulates skeletal muscle regeneration in vivo. Mol Ther 10: 844-854, 2004.

10. Bagher $\mathbf{P}$, Davis $\mathbf{M J}$, and Segal SS. Visualizing calcium responses to acetylcholine convection along endothelium of arteriolar networks in Cx40BAC-GCaMP2 transgenic mice. Am J Physiol Heart Circ Physiol 301: H794-802, 2011.

11. Bagher P, and Segal SS. Regulation of blood flow in the microcirculation: role of conducted vasodilation. Acta Physiol (Oxf) 202: 271-284, 2011. 
12. Bailey AM, O'Neill TJ, Morris CE, and Peirce SM. Arteriolar remodeling following ischemic injury extends from capillary to large arteriole in the microcirculation. Microcirculation 15: 389-404, 2008.

13. Baoge L, Van Den Steen E, Rimbaut S, Philips N, Witvrouw E, Almqvist KF, Vanderstraeten G, and Vanden Bossche LC. Treatment of skeletal muscle injury: a review. ISRN Orthop 2012: 689012, 2012.

14. Bearden SE, Payne GW, Chisty A, and Segal SS. Arteriolar network architecture and vasomotor function with ageing in mouse gluteus maximus muscle. J Physiol 561: 535-545, 2004.

15. Behringer EJ, and Segal SS. Spreading the signal for vasodilatation: implications for skeletal muscle blood flow control and the effects of ageing. J Physiol 590: 6277-6284, 2012.

16. Bentzinger CF, Wang YX, Dumont NA, and Rudnicki MA. Cellular dynamics in the muscle satellite cell niche. EMBO Rep 14: 1062-1072, 2013.

17. Berne RM. Cardiac nucleotides in hypoxia: possible role in regulation of coronary blood flow. Am J Physiol 204: 317-322, 1963.

18. Berne RM. The role of adenosine in the regulation of coronary blood flow. Circ Res 47: 807-813, 1980.

19. Bloch EH, and Iberall AS. Toward a concept of the functional unit of mammalian skeletal muscle. Am J Physiol 242: R411-420, 1982.

20. Bockman EL. Blood flow and oxygen consumption in active soleus and gracilis muscles in cats. Am J Physiol 244: H546-551, 1983.

21. Bolton TB, Lang RJ, and Takewaki T. Mechanisms of action of noradrenaline and carbachol on smooth muscle of guinea-pig anterior mesenteric artery. J Physiol 351: 549-572, 1984.

22. Borselli C, Storrie H, Benesch-Lee F, Shvartsman D, Cezar C, Lichtman JW, Vandenburgh HH, and Mooney DJ. Functional muscle regeneration with combined delivery of angiogenesis and myogenesis factors. Proc Natl Acad Sci U S A 107: 3287-3292, 2010.

23. Brock RW, Tschakovsky ME, Shoemaker JK, Halliwill JR, Joyner MJ, and Hughson RL. Effects of acetylcholine and nitric oxide on forearm blood flow at rest and after a single muscle contraction. J Appl Physiol 85: 22492254, 1998. 
24. Burton HW, and Faulkner JA. The response of arterioles in skeletal muscle grafts to vasoactive agents. Microvasc Res 34: 59-68, 1987.

25. Busse R, Edwards G, Félétou M, Fleming I, Vanhoutte PM, and Weston AH. EDHF: bringing the concepts together. Trends Pharmacol Sci 23: 374380, 2002.

26. Caldwell CJ, Mattey DL, and Weller RO. Role of the basement membrane in the regeneration of skeletal muscle. Neuropathol App/ Neurobiol 16: 225238, 1990.

27. Carlson BM, and Faulkner JA. The regeneration of skeletal muscle fibers following injury: a review. Med Sci Sports Exerc 15: 187-198, 1983.

28. Casey DP, Curry TB, and Joyner MJ. Measuring muscle blood flow: a key link between systemic and regional metabolism. Curr Opin Clin Nutr Metab Care 11: 580-586, 2008.

29. Ceafalan LC, Popescu BO, and Hinescu ME. Cellular players in skeletal muscle regeneration. Biomed Res Int 2014: 957014, 2014.

30. Chargé SB, and Rudnicki MA. Cellular and molecular regulation of muscle regeneration. Physiol Rev 84: 209-238, 2004.

31. Clifford PS. Skeletal muscle vasodilatation at the onset of exercise. J Physiol 583: 825-833, 2007.

32. Clifford PS, and Hellsten Y. Vasodilatory mechanisms in contracting skeletal muscle. J Appl Physiol 97: 393-403, 2004.

33. Clifford PS, and Jasperse JL. Feedforward vasodilatation at the onset of exercise. J Physiol 583: 811, 2007.

34. Cohen RA, and Vanhoutte PM. Endothelium-dependent hyperpolarization. Beyond nitric oxide and cyclic GMP. Circulation 92: 3337-3349, 1995.

35. Cohen RA, Weisbrod RM, Gericke M, Yaghoubi M, Bierl C, and Bolotina VM. Mechanism of nitric oxide-induced vasodilatation: refilling of intracellular stores by sarcoplasmic reticulum $\mathrm{Ca}^{2+}$ ATPase and inhibition of store-operated Ca ${ }^{2+}$ influx. Circ Res 84: 210-219, 1999.

36. Corcondilas A, Koroxenidis GT, and Shepherd JT. Effect of a brief contraction of forearm muscles on forearm blood flow J Appl Physiol 19: 142-146, 1964. 
37. Cornelison DD. Context matters: in vivo and in vitro influences on muscle satellite cell activity. J Cell Biochem 105: 663-669, 2008.

38. Cossu G, and Sampaolesi M. New therapies for Duchenne muscular dystrophy: challenges, prospects and clinical trials. Trends Mol Med 13: 520-526, 2007.

39. Costamagna D, Berardi E, Ceccarelli G, and Sampaolesi M. Adult Stem Cells and Skeletal Muscle Regeneration. Curr Gene Ther 15: 348-363, 2015.

40. Crecelius AR, Kirby BS, Luckasen GJ, Larson DG, and Dinenno FA. ATP-mediated vasodilatation occurs via activation of inwardly rectifying potassium channels in humans. J Physiol 590: 5349-5359, 2012.

41. Delashaw JB, and Duling BR. A study of the functional elements regulating capillary perfusion in striated muscle. Microvasc Res 36: 162$171,1988$.

42. Delos D, Maak TG, and Rodeo SA. Muscle injuries in athletes: enhancing recovery through scientific understanding and novel therapies. Sports Health 5: 346-352, 2013.

43. Docherty JR. Subtypes of functional $\alpha_{1}$ - and $\alpha_{2}$-adrenoceptors. Eur $J$ Pharmacol 361: 1-15, 1998.

44. Domeier TL, and Segal SS. Electromechanical and pharmacomechanical signalling pathways for conducted vasodilatation along endothelium of hamster feed arteries. J Physiol 579: 175-186, 2007.

45. Dumont NA, Bentzinger CF, Sincennes MC, and Rudnicki MA. Satellite Cells and Skeletal Muscle Regeneration. Compr Physiol 5: 1027-1059, 2015.

46. Emerson GG, and Segal SS. Alignment of microvascular units along skeletal muscle fibers of hamster retractor. J Appl Physiol 82: 42-48, 1997.

47. Faber JE. In situ analysis of $\alpha$-adrenoceptors on arteriolar and venular smooth muscle in rat skeletal muscle microcirculation. Circ Res 62: 37-50, 1988.

48. Fernando CA, Liu Y, Sowa G, and Segal SS. Attenuated rapid onset vasodilation with greater force production in skeletal muscle of caveolin-2-lmice. Am J Physiol Heart Circ Physiol 311: H415-425, 2016. 
49. Fielding RA, Manfredi TJ, Ding W, Fiatarone MA, Evans WJ, and Cannon JG. Acute phase response in exercise. III. Neutrophil and IL-1 beta accumulation in skeletal muscle. Am J Physiol 265: R166-172, 1993.

50. Forbes SJ, and Rosenthal N. Preparing the ground for tissue regeneration: from mechanism to therapy. Nat Med 20: 857-869, 2014.

51. Furchgott RF, and Zawadzki JV. The obligatory role of endothelial cells in the relaxation of arterial smooth muscle by acetylcholine. Nature 288: 373376, 1980.

52. Garland CJ, Hiley CR, and Dora KA. EDHF: spreading the influence of the endothelium. Br J Pharmacol 164: 839-852, 2011.

53. Gerhardt H. VEGF and endothelial guidance in angiogenic sprouting. Organogenesis 4: 241-246, 2008.

54. Gupta S, McArthur C, Grady C, and Ruderman NB. Stimulation of vascular $\mathrm{Na}^{+}-\mathrm{K}^{+}-$ATPase activity by nitric oxide: a cGMP-independent effect. Am J Physiol 266: H2146-2151, 1994.

55. Hamann JJ, Buckwalter JB, and Clifford PS. Vasodilatation is obligatory for contraction-induced hyperaemia in canine skeletal muscle. J Physiol 557: 1013-1020, 2004.

56. Hardy D, Besnard A, Latil M, Jouvion G, Briand D, Thépenier C, Pascal Q, Guguin A, Gayraud-Morel B, Cavaillon JM, Tajbakhsh S, Rocheteau $\mathbf{P}$, and Chrétien F. Comparative Study of Injury Models for Studying Muscle Regeneration in Mice. PLoS One 11: e0147198, 2016.

57. Helisch A, and Schaper W. Arteriogenesis: the development and growth of collateral arteries. Microcirculation 10: 83-97, 2003.

58. Hill MA, Zou H, Potocnik SJ, Meininger GA, and Davis MJ. Invited review: arteriolar smooth muscle mechanotransduction: $\mathrm{Ca}^{2+}$ signaling pathways underlying myogenic reactivity. J App/ Physiol 91: 973-983, 2001.

59. Hoier B, and Hellsten Y. Exercise-induced capillary growth in human skeletal muscle and the dynamics of VEGF. Microcirculation 21: 301-314, 2014.

60. Huard J, Li Y, and Fu FH. Muscle injuries and repair: current trends in research. J Bone Joint Surg Am 84-A: 822-832, 2002. 
61. Hudlicka $\mathbf{O}$. Microcirculation in skeletal muscle. Muscles Ligaments Tendons J 1: 3-11, 2011.

62. Ichinose E, Kurose T, Daitoku D, and Kawamata S. The skeletal muscle vascular supply closely correlates with the muscle fiber surface area in the rat. Arch Histol Cytol 71: 45-57, 2008.

63. Jackson DN, Moore AW, and Segal SS. Blunting of rapid onset vasodilatation and blood flow restriction in arterioles of exercising skeletal muscle with ageing in male mice. J Physiol 588: 2269-2282, 2010.

64. Janssen I, Heymsfield SB, Wang ZM, and Ross R. Skeletal muscle mass and distribution in 468 men and women aged 18-88 yr. J Appl Physiol 89: 81-88, 2000.

65. Järvinen TA, Järvinen TL, Kääriäinen $\mathbf{M}$, Kalimo $\mathbf{H}$, and Järvinen $\mathbf{M}$. Muscle injuries: biology and treatment. Am J Sports Med 33: 745-764, 2005.

66. Joyner MJ, and Casey DP. Regulation of increased blood flow (hyperemia) to muscles during exercise: a hierarchy of competing physiological needs. Physiol Rev 95: 549-601, 2015.

67. Karalaki M, Fili S, Philippou A, and Koutsilieris M. Muscle regeneration: cellular and molecular events. In Vivo 23: 779-796, 2009.

68. Korthuis RJ. Skeletal Muscle Circulation. Morgan \& Claypool Life Sciences San Rafael CA 142 pgs, 2011.

69. Koulmann N, Richard-Bulteau H, Crassous B, Serrurier B, Pasdeloup $\mathbf{M}$, Bigard $\mathbf{X}$, and Banzet $\mathbf{S}$. Physical exercise during muscle regeneration improves recovery of the slow/oxidative phenotype. Muscle Nerve 55: 91100, 2017.

70. Kulandavelu S, Balkan W, and Hare JM. Regulation of oxygen delivery to the body via hypoxic vasodilation. Proc Natl Acad Sci U S A 112: 62546255, 2015.

71. Latroche C, Gitiaux C, Chrétien F, Desguerre I, Mounier R, and Chazaud B. Skeletal Muscle Microvasculature: A Highly Dynamic Lifeline. Physiology (Bethesda) 30: 417-427, 2015.

72. Laumonier T, and Menetrey J. Muscle injuries and strategies for improving their repair. J Exp Orthop 3: 15, 2016. 
73. Le Moal E, Pialoux V, Juban G, Groussard C, Zouhal H, Chazaud B, and Mounier R. Redox Control of Skeletal Muscle Regeneration. Antioxid Redox Signal 27: 276-310, 2017.

74. Lee KY, Peters MC, Anderson KW, and Mooney DJ. Controlled growth factor release from synthetic extracellular matrices. Nature 408: 998-1000, 2000.

75. Lefaucheur JP, and Sébille A. The cellular events of injured muscle regeneration depend on the nature of the injury. Neuromuscul Disord 5: 501-509, 1995.

76. Levenberg S, Rouwkema J, Macdonald M, Garfein ES, Kohane DS, Darland DC, Marini R, van Blitterswijk CA, Mulligan RC, D'Amore PA, and Langer R. Engineering vascularized skeletal muscle tissue. Nat Biotechnol 23: 879-884, 2005.

77. Lu C, Zhang J, Zhang D, Uzan G, and Li M. EPCs in vascular repair: how can we clear the hurdles between bench and bedside? Front Biosci (Landmark Ed) 19: 34-48, 2014.

78. Luff SE. Ultrastructure of sympathetic axons and their structural relationship with vascular smooth muscle. Anat Embryol (Berl) 193: 515531, 1996.

79. Marshall JM. The influence of the sympathetic nervous system on individual vessels of the microcirculation of skeletal muscle of the rat. $J$ Physiol 332: 169-186, 1982.

80. Moore AW, Jackson WF, and Segal SS. Regional heterogeneity of $\alpha$ adrenoreceptor subtypes in arteriolar networks of mouse skeletal muscle. $J$ Physiol 588: 4261-4274, 2010.

81. Murrant CL. Stimulation characteristics that determine arteriolar dilation in skeletal muscle. Am J Physiol Regul Integr Comp Physiol 289: R505-R513, 2005.

82. Murrant CL, and Sarelius IH. Multiple dilator pathways in skeletal muscle contraction-induced arteriolar dilations. Am J Physiol Regul Integr Comp Physiol 282: R969-978, 2002.

83. Musaro A. The basis of muscle Regeneration. Advances in Biology: 2014, p. 16. 
84. Naik JS, Valic Z, Buckwalter JB, and Clifford PS. Rapid vasodilation in response to a brief tetanic muscle contraction. J Appl Physiol 87: 17411746, 1999.

85. Nelson CP, Rainbow RD, Brignell JL, Perry MD, Willets JM, Davies NW, Standen NB, and Challiss RA. Principal role of adenylyl cyclase 6 in $\mathrm{K}^{+}$ channel regulation and vasodilator signalling in vascular smooth muscle cells. Cardiovasc Res 91: 694-702, 2011.

86. Novielli NM, and Jackson DN. Contraction-evoked vasodilation and functional hyperaemia are compromised in branching skeletal muscle arterioles of young pre-diabetic mice. Acta Physiol (Oxf) 211: 371-384, 2014.

87. Ohyanagi M, Faber JE, and Nishigaki K. Differential activation of $\alpha_{1-}$ and $\alpha$ 2-adrenoceptors on microvascular smooth muscle during sympathetic nerve stimulation. Circ Res 68: 232-244, 1991.

88. Olfert IM, Baum O, Hellsten Y, and Egginton S. Advances and challenges in skeletal muscle angiogenesis. Am J Physiol Heart Circ Physiol 310: H326-336, 2016.

89. Organization WH. Global Status Report on Road Safety World Health Organization 340, 2015.

90. Peirce SM, and Skalak TC. Microvascular remodeling: a complex continuum spanning angiogenesis to arteriogenesis. Microcirculation 10: 99-111, 2003.

91. Price RJ, and Skalak TC. Arteriolar remodeling in skeletal muscle of rats exposed to chronic hypoxia. J Vasc Res 35: 238-244, 1998.

92. Prisk V, and Huard J. Muscle injuries and repair: the role of prostaglandins and inflammation. Histol Histopathol 18: 1243-1256, 2003.

93. Quarta M, Cromie M, Chacon R, Blonigan J, Garcia V, Akimenko I, Hamer M, Paine P, Stok M, Shrager JB, and Rando TA. Bioengineered constructs combined with exercise enhance stem cell-mediated treatment of volumetric muscle loss. Nat Commun 8: 15613, 2017.

94. Richard-Bulteau H, Serrurier B, Crassous B, Banzet S, Peinnequin A, Bigard X, and Koulmann N. Recovery of skeletal muscle mass after extensive injury: positive effects of increased contractile activity. Am J Physiol Cell Physiol 294: C467-476, 2008. 
95. Rigamonti E, Touvier T, Clementi E, Manfredi AA, Brunelli S, and Rovere-Querini P. Requirement of inducible nitric oxide synthase for skeletal muscle regeneration after acute damage. J Immunol 190: 17671777, 2013.

96. Rogers R, Baumann C, and Otis J. Recovery of skeletal muscle function following injury is not augmented by acute resveratrol supplementation. Int J Clin Exp Physiol: 2015, p. 29-33.

97. Ross GA, Mihok ML, and Murrant CL. Extracellular adenosine initiates rapid arteriolar vasodilation induced by a single skeletal muscle contraction in hamster cremaster muscle. Acta Physiol (Oxf) 208: 74-87, 2013.

98. Ruhrberg C. Growing and shaping the vascular tree: multiple roles for VEGF. Bioessays 25: 1052-1060, 2003.

99. Saclier M, Cuvellier S, Magnan M, Mounier R, and Chazaud B. Monocyte/macrophage interactions with myogenic precursor cells during skeletal muscle regeneration. FEBS J 280: 4118-4130, 2013.

100. Saltin B, Rådegran G, Koskolou MD, and Roach RC. Skeletal muscle blood flow in humans and its regulation during exercise. Acta Physiol Scand 162: 421-436, 1998.

101. Sarelius I, and PohI U. Control of muscle blood flow during exercise: local factors and integrative mechanisms. Acta Physiol (Oxf) 199: 349-365, 2010.

102. Sato A, Terata K, Miura H, Toyama K, Loberiza FR, Hatoum OA, Saito T, Sakuma I, and Gutterman DD. Mechanism of vasodilation to adenosine in coronary arterioles from patients with heart disease. Am J Physiol Heart Circ Physiol 288: H1633-1640, 2005.

103. Seaman SA, Cao Y, Campbell CA, and Peirce SM. Macrophage Recruitment and Polarization During Collateral Vessel Remodeling in Murine Adipose Tissue. Microcirculation 23: 75-87, 2016.

104. Segal SS. Integration and Modulation of Intercellular Signaling Underlying Blood Flow Control. J Vasc Res 52: 136-157, 2015.

105. Segal SS. Regulation of blood flow in the microcirculation. Microcirculation 12: 33-45, 2005.

106. Segal SS, Faulkner JA, and White TP. Skeletal muscle fatigue in vitro is temperature dependent. J Appl Physiol 61: 660-665, 1986. 
107. Segal SS, and Kurjiaka DT. Coordination of blood flow control in the resistance vasculature of skeletal muscle. Med Sci Sports Exerc 27: 11581164, 1995.

108. Segal SS, White TP, and Faulkner JA. Architecture, composition, and contractile properties of rat soleus muscle grafts. Am J Physiol 250: C474479, 1986.

109. Shoemaker JK, Tschakovsky ME, and Hughson RL. Vasodilation contributes to the rapid hyperemia with rhythmic contractions in humans. Can J Physiol Pharmacol 76: 418-427, 1998.

110. Sinkler SY, Fernando CA, and Segal SS. Differential $\alpha$-adrenergic modulation of rapid onset vasodilatation along resistance networks of skeletal muscle in old versus young mice. J Physiol 594: 6987-7004, 2016.

111. Sinkler SY, and Segal SS. Aging alters reactivity of microvascular resistance networks in mouse gluteus maximus muscle. Am J Physiol Heart Circ Physiol 307: H830-839, 2014.

112. Sinkler SY, and Segal SS. Rapid versus slow ascending vasodilatation: intercellular conduction versus flow-mediated signalling with tetanic versus rhythmic muscle contractions. J Physiol 595: 7149-7165, 2017.

113. Smith C, Kruger MJ, Smith RM, and Myburgh KH. The inflammatory response to skeletal muscle injury: illuminating complexities. Sports Med 38: 947-969, 2008.

114. Somlyo AP, and Somlyo AV. $\mathrm{Ca}^{2+}$ sensitivity of smooth muscle and nonmuscle myosin II: modulated by $G$ proteins, kinases, and myosin phosphatase. Physiol Rev 83: 1325-1358, 2003.

115. Somlyo AP, and Somlyo AV. Signal transduction and regulation in smooth muscle. Nature 372: 231-236, 1994.

116. Stark DA, Karvas RM, Siegel AL, and Cornelison DD. Eph/ephrin interactions modulate muscle satellite cell motility and patterning. Development 138: 5279-5289, 2011.

117. Tatsumi R, Liu X, Pulido A, Morales M, Sakata T, Dial S, Hattori A, Ikeuchi $\mathbf{Y}$, and Allen RE. Satellite cell activation in stretched skeletal muscle and the role of nitric oxide and hepatocyte growth factor. Am J Physiol Cell Physiol 290: C1487-1494, 2006. 
118. Tidball JG. Inflammatory processes in muscle injury and repair. Am J Physiol Regul Integr Comp Physiol 288: R345-353, 2005.

119. Tidball JG. Mechanisms of muscle injury, repair, and regeneration. Compr Physiol 1: 2029-2062, 2011.

120. Tidball JG. Regulation of muscle growth and regeneration by the immune system. Nat Rev Immunol 17: 165-178, 2017.

121. Toumi $\mathbf{H}$, and Best TM. The inflammatory response: friend or enemy for muscle injury? Br J Sports Med 37: 284-286, 2003.

122. Tschakovsky ME, and Sheriff DD. Immediate exercise hyperemia: contributions of the muscle pump vs. rapid vasodilation. J Appl Physio/ 97: 739-747, 2004.

123. Tschakovsky ME, Shoemaker JK, and Hughson RL. Vasodilation and muscle pump contribution to immediate exercise hyperemia. Am J Physiol 271: H1697-1701, 1996.

124. van Hinsbergh VW, and Koolwijk P. Endothelial sprouting and angiogenesis: matrix metalloproteinases in the lead. Cardiovasc Res 78: 203-212, 2008.

125. VanTeeffelen JW, and Segal SS. Effect of motor unit recruitment on functional vasodilatation in hamster retractor muscle. J Physiol $524 \mathrm{Pt} 1$ : 267-278, 2000.

126. VanTeeffelen JW, and Segal SS. Rapid dilation of arterioles with single contraction of hamster skeletal muscle. Am J Physiol Heart Circ Physiol 290: H119-127, 2006.

127. Winkler T, von Roth P, Matziolis G, Schumann MR, Hahn S, Strube $\mathbf{P}$, Stoltenburg-Didinger G, Perka C, Duda GN, and Tohtz SV. Time course of skeletal muscle regeneration after severe trauma. Acta Orthop 82: 102111, 2011.

128. Wunsch SA, Muller-Delp J, and Delp MD. Time course of vasodilatory responses in skeletal muscle arterioles: role in hyperemia at onset of exercise. Am J Physiol Heart Circ Physiol 279: H1715-1723, 2000.

129. Yardeni T, Eckhaus M, Morris HD, Huizing M, and Hoogstraten-Miller S. Retro-orbital injections in mice. Lab Anim (NY) 40: 155-160, 2011. 
130. Zhang M, Malik AB, and Rehman J. Endothelial progenitor cells and vascular repair. Curr Opin Hematol 21: 224-228, 2014.

131. Zisch AH, Lutolf MP, and Hubbell JA. Biopolymeric delivery matrices for angiogenic growth factors. Cardiovasc Pathol 12: 295-310, 2003. 
Charmain was born and raised in Colombo, Sri Lanka. She arrived in the United States in 2004 for her undergraduate studies after completing her high school education at Holy Family Convent, Colombo, Sri Lanka in 2003. She completed her bachelor's degree at Wilson College in Chambersburg, Pennsylvania in 2008, and soon after graduation she worked at the University of California, Los Angeles, as a research assistant for a year between 2008 and 2009 before pursuing postgraduate studies. She completed a master's degree in 2013 at the University of Central Arkansas in Conway, Arkansas under the supervision of Prof. Brent Hill before pursuing a PhD at the University of Missouri, Columbia, Missouri under the supervision of Prof. Steven Segal. Throughout her tenure as a PhD student, Charmain's research has been supported by a teaching fellowship from the University of Missouri, a Margaret Proctor Mulligan Fellowship and by a Predoctoral Fellowship 17PRE33410260 from the Midwest Affiliate of the American Heart Association. 